\title{
Contextual Semantics in Quantum Mechanics from a Categorical Point of View
}

\author{
Vassilios Karakostas and Elias Zafiris
}

\begin{abstract}
The category-theoretic representation of quantum event structures provides a canonical setting for confronting the fundamental problem of truth valuation in quantum mechanics as exemplified, in particular, by Kochen-Specker's theorem. In the present study, this is realized on the basis of the existence of a categorical adjunction between the category of sheaves of variable local Boolean frames, constituting a topos, and the category of quantum event algebras. We show explicitly that the latter category is equipped with an object of truth values, or classifying object, which constitutes the appropriate tool for assigning truth values to propositions describing the behavior of quantum systems. Effectively, this category-theoretic representation scheme circumvents consistently the semantic ambiguity with respect to truth valuation that is inherent in conventional quantum mechanics by inducing an objective contextual account of truth in the quantum domain of discourse. The philosophical implications of the resulting account are analyzed. We argue that it subscribes neither to a pragmatic instrumental nor to a relative notion of truth. Such an account essentially denies that there can be a universal context of reference or an Archimedean standpoint from which to evaluate logically the totality of facts of nature.
\end{abstract}

1. Introduction: Modus Operandi of the Traditional Scheme of Correspondence TruthSemantics in Physical Science

2. Propositional Truth in Standard Quantum Mechanics

3. Motivating the Application of the Categorical Framework to Quantum Semantics

4. Category-Theoretic Representation of Quantum Event Algebras

\subsection{Conceptual Considerations}

4.2 Functorial Modeling of Quantum Event Algebras

5. Equivalence Classes of Pointed Boolean Frames

6. Boolean Localization Functors

7. Existence of the Subobject Classifier in $\mathcal{L}$

7.1 The Quantum Object of Truth Values

8. Quantum Logical Structure of Truth Values and Criterion of Truth

9. Contextual Semantics of Quantum Truth Valuation: Conceptual and Philosophical Implications

10. Appendix

\footnotetext{
${ }^{0}$ To contact the authors, please write to: Vassilios Karakostas, Department of Philosophy and History of Science, Faculty of Sciences, University of Athens, Athens 157 71, Greece; e-mail: karakost@phs.uoa.gr, or to Elias Zafiris, Department of Logic, Eötvös University, Budapest, Hungary; e-mail: ezafiris@math.uoa.gr.
} 


\section{Introduction: Modus Operandi of the Traditional Scheme of Correspondence Truth-Semantics in Physical Science}

The logic of a physical theory reflects the structure of elementary propositions referring to the behavior of a physical system in the domain of the corresponding theory. Elementary propositions concern the values of physical quantities pertaining to a system and are of the form "the value of the physical quantity $A$ lies in a measurable subset of the real numbers", for which we use the shorthand notation " $A \in \Delta$ ". Propositions of the type " $A \in \Delta$ " refer to the world in the most direct conceivable sense. They can only be ascertained as true or false by corresponding, or not corresponding, to "facts" or, more generally, to "actual states of affairs" or, in contemporary physical terms, to "events" conceived in the physicist's understanding as actual measurement outcomes, as concrete elements of empirical reality (e.g., Svozil 1998). In this respect, elementary propositions are posits in the process of probing the natural world. They can be used to indicate properties of physical systems in the following sense: a system $S$ is characterized by a property $a$, namely a particular value of the physical quantity $A$, if and only if the corresponding proposition $P_{A}$ is true, i.e., if and only if " $A=a$ " or " $A \in \Delta$ ". For instance, " $q_{x}=5$ " is true of the system $S$ if and only if the $x$ component of $S$ 's position is 5 according to a proper system of coordinates. To say, therefore, that the elementary proposition " $A \in \Delta$ " is true of the system $S$ is equivalent to say that in $S, A$ has a value in $\Delta$. Physics is fundamentally concerned about the truth values of such propositions of the form " $A \in \Delta$ ", where $\Delta$ varies over the Borel subsets of the real line and $A$ varies over the physical quantities of system $S$, when $S$ is in a given state. Of course, the state may change in time, and hence the truth values also will change in time.

In addition to the syntactic aspect, dealing with the logico-mathematical operations and relations among the propositions pertaining to a system, the logic of a physical theory incorporates the task of providing a semantics, focusing particularly on the task of establishing a criterion of truth or, in a more complete sense, defining propositional truth. In relation to the latter, the correspondence theory of truth has frequently been regarded within physical science as the most eminent. Although the correspondence theory admits various different formulations, the core of any correspondence theory is the idea that a proposition is true if and only if it corresponds to or matches reality. The classical version of the theory describes this relationship as a correspondence to the facts about the world (e.g., Burgess et al. 2011, pp. 70-72). If so, then adopting a correspondence theory of truth amounts to endorsing instances of the following scheme:

[CF] The proposition that $P$ is true if and only if $P$ corresponds to a fact.

Alternatively, if one construes the notion of a "fact" in terms of the weaker notion of an obtaining "state of affairs", as in an Austin-type theory, ${ }^{1}$ then, $[\mathrm{CF}]$ is re-expressed as follows:

[CS] The proposition that $P$ is true if and only if there is a state of affairs $X$ such that

$P$ corresponds to $X$ and $X$ obtains.

The useful feature of states of affairs, especially in the philosophical discourse, is that they refer to something that can be said to obtain or fail to obtain, to be the case or not to be the case, to be a fact or fail to be a fact, that is, they exist even when they are not concretely manifested or realized.

It is worthy to note in this connection that the traditional theory of truth as correspondence, regardless of its exact formulation, has frequently been associated with the view that truth is radically non-epistemic (e.g., Devitt 2001, p. 606). This means that the truth (or falsity) of a proposition is entirely independent of anyone's cognitive capacities, beliefs, theories,

\footnotetext{
${ }^{1}$ Austin's account of a correspondence theory of truth is reprinted, among other classical essays concerning the nature of truth, in Blackburn and Simmons (1999, pp. 149-162).
} 
conceptual schemes, and so on. According to this non-epistemic conception, the truthmakers of propositions - namely, facts or actual states of affairs, forming the object-end of the truthmaking relation - are totally independent of human conceptualization and thus can be conceived of as being completely autonomous in themselves, or as residing in the world purely extensionally, that is, in a manner independent of our worldviews and particular discursive practices and contexts. In this sense, the view of a radically non-epistemic conception of truth incorporates the following transcendence condition:

Transcendence condition: The truth of a proposition transcends our possible knowledge of it, or its evidential basis; it is empirically unconstrained.

As a consequence of the preceding condition, even if it is impossible to produce a framework on which we may ascertain the truth value of a proposition this does not imply that the proposition does not possess any such value. It always has one. It is either determinately true or determinately false independently of any empirical evidence or cognitive means by which we may establish which value it is. The possession of truth values is therefore entirely independent of our means of warranting their assignment.

The transcendence condition, no doubt, attempts to capture the realist intuition that a proposition cannot be claimed true or false in virtue of its knowability or justifiability. For, a proposition may be true without being justified, and vice versa. Agreed! But what does the transcendence thesis, in its totality, really presuppose, especially when viewed within the traditional framework of correspondence truth? It presupposes the existence of a 'platonic' universe of true propositions, entirely independent of our ability in having access to it, and, henceforth, elements of this ideal world, in this case, propositions, possess determinate truth values entirely independent of our capability in forming justified convictions about them. In other words, it is important to realize that this thesis does not simply aim to establish an objective basis or attribute a non-epistemic character to the notion of truth - that, for instance, the content of declarative propositions is rendered true (or false) on the basis of worldly conditions and not on some relevant beliefs of ours - but this particular conception tends to be so radically non-epistemic that at the end leads to a notion of truth with absolutely no epistemic features.

Be that as it may, it is particularly interesting that the semantics underlying the propositional structure of classical mechanics allows truth-value assignment in conformity with the usual traditional conception of a correspondence account of truth. In classical physics, the algebra of propositions of a classical system is isomorphic to the lattice of subsets of phase space, a Boolean lattice, $L_{B}$, that can be interpreted semantically by a two-valued truthfunction. This means that to every proposition $P \in L_{B}$ one of the two possible truth values 1 (true) and 0 (false) can be assigned; accordingly, any proposition is either true or false (tertium non datur) (e.g., Dalla Chiara et al. 2004, p. 21). Specifically, each point in phase space, representing a classical state of a given system $S$, defines a truth-value assignment to the subsets representing the propositions. Each subset to which the point belongs represents a true proposition or a property that is instantiated by the system. Likewise, each subset to which the point does not belong represents a false proposition or a property that is not instantiated by the system. Thus, every possible property of $S$ is selected as either occurring or not; equivalently, every corresponding proposition pertaining to $S$ is either true or false. Henceforth, all propositions of a classical system are semantically decidable.

From a physical point of view, this is immediately linked to the fact that classical physics views objects-systems as bearers of determinate properties. That is, properties of classical systems are considered as being intrinsic to the system; they are independent of whether or not any measurement is attempted on them and their definite values are independent of one another as far as measurement is concerned. All properties pertaining to a classical system are simultaneously determinate and potentially available to the system, independently of any perceptual evidence or cognitive means by which we may verify or falsify them. Accordingly, 
the propositions of a classical system are considered as possessing determinate truth values - they are either determinately true or determinately false - prior to and independent of any actual investigation of the states of affairs the propositions denote; that is, classical mechanical propositions possess investigation-independent truth values, thus capturing the radically non-epistemic character of a traditional correspondence account of truth (Karakostas 2012). Consequently, since in a classical universe of discourse all propositions are meant to have determinate truth-conditions, there is supposed to exist implicitly an Archimedean standpoint from which the totality of facts may be logically evaluated.

\section{Propositional Truth in Standard Quantum Mechanics}

In contradistinction to the Boolean propositional structure of classical mechanics, the logical structure of a quantum system is neither Boolean nor possible to be embedded into a Boolean lattice. On the standard (Dirac-von Neumann) codification of quantum theory, the elementary propositions pertaining to a system form a non-Boolean lattice, $L_{H}$, isomorphic to the lattice of closed linear subspaces or corresponding projection operators of a Hilbert space. Thus, a proposition pertaining to a quantum system is represented by a projection operator $P$ on the system's Hilbert space $H$, or equivalently, it is represented by the linear subspace $H_{P}$ of $H$ upon which the projection operator $P$ projects (e.g., Rédei 1998, Section 4.2). Since each projection operator $P$ on $H$ acquires two eigenvalues 1 and 0 , where the value 1 can be read as "true" and 0 as "false", the proposition asserting that "the value of the physical quantity $A$ of system $S$ lies in a certain range of values $\Delta$ ", or equivalently, that "system $S$ in state $|\psi\rangle$ acquires the property $P(A)$ ", is said to be true if and only if the corresponding projection operator $P_{A}$ obtains the value 1 , that is, if and only if $P_{A}|\psi\rangle=|\psi\rangle$. Accordingly, the state $|\psi\rangle$ of the system lies in the associated subspace $H_{A}$ which is the range of the operator $P_{A}$, i.e., $|\psi\rangle \in H_{A}$. In such a circumstance, the property $P(A)$ pertains to the quantum system $S$. Otherwise, if $P_{A}|\psi\rangle=0$ and, hence, $|\psi\rangle \in \perp H_{A}$ (subspace completely orthogonal to $H_{A}$ ), the counter property $\neg P(A)$ pertains to $S$, and the proposition is said to be false. It might appear, therefore, that propositions of this kind have a well-defined truth value in a sense analogous to the truth-value assignment in classical mechanics.

There is, however, a significant difference between the two situations. Unlike the case in classical mechanics, for a given quantum system, the propositions represented by projection operators or Hilbert space subspaces are not partitioned into two mutually exclusive and collectively exhaustive sets representing either true or false propositions. As already pointed out, only propositions represented by subspaces that contain the system's state are assigned the value "true" (propositions assigned probability 1 by $|\psi\rangle$ ), and only propositions represented by spaces orthogonal to the state are assigned the value "false" (propositions assigned probability 0 by $|\psi\rangle$ ) (Dirac 1958, pp. 46-47; von Neumann 1955, pp. 213-217). Hence, propositions represented by subspaces that are at some non-zero or non-orthogonal angle to the unit vector $|\psi\rangle$ or, more appropriately, to the ray representing the quantum state are not assigned any truth value in $|\psi\rangle$. These propositions are neither true nor false; they are assigned by $|\psi\rangle$ a probability value different from 1 and 0 ; thus, they are undecidable or indeterminate for the system in state $|\psi\rangle$ and the corresponding properties are taken as indefinite.

This kind of semantic indeterminacy imposes an inherent ambiguity with respect to the classical binary true/false value assignments rigorously expressed, for the first time, by Kochen-Specker's (1967) theorem. According to this, for any quantum system associated to a Hilbert space of dimension higher than two, there does not exist a two-valued, truth-functional assignment $h: L_{H} \rightarrow\{0,1\}$ on the set of closed linear subspaces, $L_{H}$, interpretable as quantum mechanical propositions, preserving the lattice operations and the orthocomplement. In other words, the gist of the theorem, when interpreted semantically, asserts the impossibility of assigning definite truth values to all propositions pertaining to a physical system at any one time, for any of its quantum states, without generating a contradiction. 


\section{Motivating the Application of the Categorical Framework to Quantum Semantics}

It should be underlined, however, that although the preceding Kochen-Specker result forbids a global, absolute assignment of truth values to quantum mechanical propositions, it does not exclude ones that are contextual (Karakostas 2007). Here, "contextual" means that the truth value given to a proposition depends on which subset of mutually commuting projection operators (meaning "simultaneously measurable") one may consider it to be a member, i.e., it depends on which other compatible propositions are given truth values at the same time. To explicate this by means of a generalized example, let $A, B$ and $E$ denote observables of the same quantum system $S$, so that the corresponding projection operator $A$ commutes with operators $B$ and $E([A, B]=0=[A, E])$, not however the operators $B$ and $E$ with each other $([B, E] \neq 0)$. Then, due to the incompatibility of the last pair of observables, the result of a measurement of $A$ depends on whether the system had previously been subjected to a measurement of the observable $B$ or a measurement of the observable $E$ or in none of them. Thus, the value of the observable $A$ depends upon the set of mutually commuting observables one may consider it with, that is, the value of $A$ depends upon the selected set of measurements. In other words, the value of the observable $A$ cannot be thought of as prefixed, as being independent of the experimental context actually chosen, as specified, in our example, by the $\{A, B\}$ or $\{A, E\}$ frame of mutually compatible observables. Accordingly, the truth value assigned to the associated proposition " $A \in \Delta$ " — i.e., "the value of the observable $A$ of system $S$ lies in a certain range of values $\Delta$ " - should be contextual as it depends on whether $A$ is thought of in the context of simultaneously ascribing a truth value to propositions about $B$, or to propositions about $E$. Of course, the formalism of quantum theory does not imply how such a contextual valuation might be obtained, or what properties it should possess. To this end, we resort to the powerful methods of categorical topos theory, which directly captures the idea of structures varying over contexts, thus providing a natural setting for studying contextuality phenomena (e.g., Abramsky and Brandenburger 2011), providing consistently context-dependent operations in logic (e.g., Domenech and Freytes 2005), representing various physical processes (e.g., Coecke 2010), and even motivating a contemporary contemplation of Whitehead's process philosophy (Epperson and Zafiris 2013).

In this work, the research path we propose implements the intuitively clear idea of probing the global structure of a quantum algebra of events (or propositions) in terms of structured multitudes of interlocking local Boolean frames. It is probably one of the deepest insights of modern quantum theory that whereas the totality of all experimental/empirical facts can only be represented in a globally non-Boolean logical structure, the acquisition of every single fact depends on a locally Boolean context. Indeed, we view each preparatory Boolean environment of measurement as a context that offers a 'classical perspective' on a quantum system. A classical perspective or context is nothing but a set of commuting physical quantities, as in the preceding example, or, more precisely, a complete Boolean algebra of commuting projection operators generated by such a set. Physical quantities in any such algebra can be given consistent values, as in classical physics. Thus, each context functions as a Boolean frame providing a local 'classical' viewpoint on reality. No single context or perspective can deliver a complete picture of the quantum system, but, by applying category-theoretic reasoning, it is possible to use the collection of all of them in an overall structure that will capture the entire system. It is also of great importance how the various contexts relate to each other. Categorically speaking, this consideration is naturally incorporated into our scheme, since the category-theoretic representation of quantum event structures in terms of Boolean localization contexts can be described by means of a topos, which stands for a category of sheaves of variable local Boolean frames encoding the global logical information of these localization contexts.

In a well defined sense, topos theory provides us with the first natural examples of global multi-valued functional truth structures. By definition, a topos, conceived as a category of 
sheaves for a categorical topology, is equipped with an internal object of truth values, called a subobject classifier, which generalizes the classical binary object of truth values used for valuations of propositions (Lawvere and Schanuel 2009, pp. 338-343). As explained below, this generalized object of truth values in a topos is not ad hoc, but reflects genuine constraints of the surrounding universe of discourse. We will show, in particular, that the topos-theoretic representation scheme of quantum event algebras by means of variable local Boolean frames induces an object of truth values, or classifying object, which constitutes the appropriate tool for the definition of quantum truth-value assignments, corresponding to valuations of propositions describing the behavior of quantum systems. This, in effect, characterizes the novelty of our approach and its fruitfulness for a consistent contextual account of truth in the quantum domain in comparison to a multiplicity of various other approaches on the foundations of quantum physics.

\section{Category-Theoretic Representation of Quantum Event Algebras}

As indicated in the preceding section, the global semantic ambiguity of the non-Boolean logical structure of quantum mechanics, expressed formally by Kochen-Specker's theorem, does not exclude local two-valued truth-functional assignments with respect to complete Boolean algebras of projection operators on the Hilbert space of a quantum system. More precisely, each self-adjoint operator representing an observable has associated with it a Boolean subalgebra which is identified with the Boolean algebra of projection operators belonging to its spectral decomposition. Hence, given a set of observables of a quantum system, there always exists a complete Boolean algebra of projection operators, namely, a local Boolean subalgebra of the global non-Boolean event algebra of a quantum system with respect to which a local two-valued truth-functional assignment is meaningful, if and only if the given observables are simultaneously measurable. Consequently, the possibility of local two-valued truth-functional assignments of the global non-Boolean event algebra of a quantum system points to the assumption that complete Boolean algebras play the role of local Boolean logical frames for contextual true/false value assignments. The modeling scheme we propose in order to implement this idea in a universal way, so that the global structure of a quantum system to be modeled categorically in terms of a topos of sheaves of local Boolean frames, uses the technical apparatus of categorical sheaf theory (Mac Lane and Moerdijk 1992, Awodey 2010). The basic conceptual steps involved in the realization of the suggested approach may be summarized as follows.

\subsection{Conceptual considerations}

Firstly, we introduce the notion of a topological covering scheme of a quantum event algebra (Zafiris 2006a) consisting of epimorphic families of local Boolean logical frames. From the physical point of view, this attempt amounts to analyze or 'co-ordinatize' the information contained in a quantum event algebra $L$ by means of structure preserving morphisms $B \rightarrow L$, having as their domains locally defined Boolean event algebras $B$. Any single map from a Boolean domain to a quantum event algebra does not suffice for a complete determination of the latter's information content, and thus, it contains only a limited amount of information about it. Specifically, it includes only the amount of information related to a Boolean reference context and inevitably is constrained to represent exclusively the abstractions associated with it. This problem is confronted by employing a sufficient amount of maps, organized in terms of covering sieves, from the coordinatizing Boolean domains to a quantum event algebra so as to cover it completely. These maps play exactly the role of local Boolean covers for the filtration of the information associated with a quantum structure of events, in that, their domains $B$ provide Boolean coefficients associated with typical measurement situations of 
quantum observables. The local Boolean covers capture individually complementary features of a quantum algebra of events and provide collectively its categorical local decomposition in the descriptive terms of Boolean reference frames. Technically, this is described by an action of a category of local Boolean frames on a global quantum event algebra, forming a presheaf.

Secondly, we define appropriate compatibility conditions between overlapping local Boolean covers. This is necessary since it enforces an efficient, uniquely defined pasting code between different local covers of a quantum algebra of events. Technically, this is described by the notion of a Boolean localization functor, or equivalently, by a structure sheaf of Boolean coefficients of a quantum event algebra. Category-theoretically, one may think of a Boolean localization functor as consisting of epimorphic families or diagrams of Boolean covers interconnected together targeting a quantum event algebra.

Thirdly, we establish the necessary and sufficient conditions for the isomorphic representation of quantum event algebras in terms of Boolean localization functors. The crucial technical and semantical method used in order to establish these conditions is based on the existence of a categorical adjunction, consisting of a pair of adjoint functors, between the category of (pre)sheaves of variable local Boolean frames and the category of quantum event algebras. In essence, this Boolean frames-quantum adjunction provides a bidirectional and functorial translational mechanism of encoding and decoding information between the Boolean and quantum kind of structures respecting their distinctive form. It is precisely the existence of this categorical adjunction that allows the isomorphic representation of quantum event algebras in terms of sheaves of variable local Boolean frames.

The notion of a sheaf incorporates the requirements of consistency under extension from the local Boolean to the global quantum level, and inversely, under restriction of the global quantum to the local Boolean level. The functional dependence implicated by a categorical sheaf relativizes the presupposed rigid relations between quantum events with respect to variable local Boolean frames conditioning the actualization of events. The category of sheaves of variable local Boolean frames, encoding the global logical information of Boolean localization functors, constitutes a topos providing the possibility of applying the powerful logical classification methodology of topos theory with reference to the quantum universe of discourse.

Topos theoretical approaches to the logical foundations of quantum mechanics have also been considered, from a different viewpoint, by Isham and Butterfield (1998), Döring and Isham (2011), Abramsky and Brandenburger (2011), Heunen, Landsmann and Spitters (2011), van den Berg and Heunen (2012). In Zafiris and Karakostas (2013) we compare each one of the preceding approaches with our topos-theoretic representation scheme of quantum event algebras. In particular, by restricting considerations for present purposes to the notion of subobject classifier, functioning as an object of truth values or classifying object, these approaches assume axiomatically the existence of a partially ordered set of Boolean contexts, construct the associated category of presheaves on this partial order, and make use of the subobject classifier of this presheaf topos for truth valuations. On the contrary, in the present work, the full category of complete Boolean algebras with Boolean homomorphisms is used as a category of possible frames (by virtue of the spectral theorem) without any restriction to inclusions or injective morphisms. Moreover, the subobject classifier for quantum truth valuations is not the presheaf subobject classifier, but it is naturally induced, as already pointed out, via a pair of adjoint functors between the category of quantum event algebras and the category of sheaves over the above Boolean frames. In this manner, from a logical perspective, the aforementioned categorical adjunction plays the role of a semantic bridge between these two categories effecting the filtration and localization of quantum truth valuations through the underlying Boolean frames using topos-theoretic arguments. We further note in this connection that of particular relevance to the present work, regarding the specification of an object of truth values, although not based on categorical methods, appears to be the approach to the foundations of quantum logic by Davis (1977) and Takeuti (1978), according to whom, 
'quantization' of a proposition of classical physics is equivalent to interpreting it in a Boolean extension of a set theoretical universe, where $B$ is a complete Boolean algebra of projection operators on a Hilbert space.

It is not possible to provide here a concise account of category theory. For a general introduction to this well-developed mathematical framework, topos theory and categorial logic, the reader may consult Lawvere and Schanuel (2009), Bell (1988/2008) and Goldblatt $(1984 / 2006)$. For the convenience of the reader, we limit ourselves to summarize in the appendix certain aspects of category theory used systematically in the present work. In the following subsection, we define the basic categorical structures involved in the functorial representation of a quantum event algebra as a topos of (pre)sheaves over the base category of Boolean event algebras.

\subsection{Functorial Modeling of Quantum Event Algebras}

A Boolean categorical event structure is a small category, denoted by $\mathcal{B}$, which is called the category of Boolean event algebras. The objects of $\mathcal{B}$ are $\sigma$-Boolean algebras of events and the arrows are the corresponding Boolean algebraic homomorphisms.

A quantum categorical event structure is a locally small co-complete category, denoted by $\mathcal{L}$, which is called the category of quantum event algebras. The objects of $\mathcal{L}$ are quantum event algebras and the arrows are quantum algebraic homomorphisms. A quantum event algebra $L$ in $\mathcal{L}$ is defined as an orthomodular $\sigma$-orthoposet (Dalla Chiara et al. 2004, Zafiris 2006a), that is, as a partially ordered set of quantum events, endowed with a maximal element 1 , and with an operation of orthocomplementation $[-]^{*}: L \rightarrow L$, which satisfy, for all $l \in L$, the following conditions: [i] $l \leq 1$, [ii] $l^{* *}=l$, [iii] $l \vee l^{*}=1$, [iv] $l \leq \dot{l} \Rightarrow l^{*} \leq l^{*}$, [v] $l \perp \hat{l} \Rightarrow l \vee i \in L$, [vi] for $l, \hat{l} \in L, l \leq \hat{l}$ implies that $l$ and $l$ are compatible, where $0:=1^{*}, l \perp \dot{l}:=l \leq l^{*}$, and the operations of meet $\wedge$ and join $\vee$ are defined as usually. The $\sigma$-completeness condition, meaning that the join of countable families of pairwise orthogonal events exists, is required in order to have a well defined theory of quantum observables over $L$.

If $L$ is isomorphic with the orthocomplemented lattice of orthogonal projections on a complex Hilbert space, then from von Neumann's spectral theorem the quantum observables are in bijective correspondence with the Hermitian operators on the Hilbert space. As noted in Section 2, the original quantum logical formulation of quantum theory depends in an essential way on the identification of propositions with projection operators on a complex Hilbert space. In the sequel, the measure-theoretic $\sigma$-completeness condition is not going to play any particular role in the exposition of the arguments, so one may drop it and consider complete Boolean algebras and complete orthomodular lattices instead.

The crucial observation pointing to the significance of the proposed functorial modeling approach to quantum event algebras is that despite the fact that each object $L$ in $\mathcal{L}$ is not Boolean, there always exists an underlying categorical diagram of Boolean subalgebras of $L$, where each one of them is generated by the following well-known compatibility condition: any arbitrary pair of elements $l, \hat{l} \in L$ are compatible if the sublattice generated by $\left\{l, l^{*}, l_{,} l^{*}\right\}$ is a Boolean algebra, namely, if it is a Boolean sublattice. Thus, if these Boolean sublattices of events can be appropriately identified in terms of local Boolean logical frames of a quantum event algebra, then the global information of the latter can be recovered in a structurepreserving way by a suitable sheaf-theoretic construction gluing together categorical diagrams of local Boolean subobjects.

A topological gluing construction of this form can only take place in an extension of $\mathcal{B}$ enunciating the free completion of $\mathcal{B}$ under colimits. Technically, this process requires initially the construction of the functor category of presheaves of sets on Boolean event algebras, denoted by $\operatorname{Sets}^{\mathcal{B}^{\text {op }}}$, where $\mathcal{B}^{o p}$ is the opposite category of $\mathcal{B}$. The objects of Sets $^{\mathcal{B}^{o p}}$ are all functors $\mathbf{P}: \mathcal{B}^{o p} \rightarrow$ Sets, and morphisms all natural transformations between such functors. Each object $\mathbf{P}$ in the category of presheaves $\mathbf{S e t s}^{\mathcal{B}^{o p}}$ is a contravariant set-valued functor on $\mathcal{B}$, called a presheaf on $\mathcal{B}$. The functor category of presheaves on Boolean event algebras, 
Sets $^{\mathcal{B}^{o p}}$, provides the instantiation of a structure known as topos. A topos exemplifies a well-defined notion of a universe of variable sets. It can be conceived as a local mathematical framework corresponding to a generalized model of set theory or as a generalized topological space.

For each Boolean algebra $B$ of $\mathcal{B}$, a presheaf $\mathbf{P}$ on $B$, denoted by $\mathbf{P}(\mathrm{B})$, is a set, and for each Boolean homomorphism $f: C \rightarrow B, \mathbf{P}(f): \mathbf{P}(B) \rightarrow \mathbf{P}(\mathrm{C})$ is a set-theoretic function such that, if $p \in \mathbf{P}(\mathrm{B})$, the value $\mathbf{P}(f)(p)$ for an arrow $f: C \rightarrow B$ in $\mathcal{B}$ is called the restriction of $p$ along $f$ and is denoted by $\mathbf{P}(f)(p)=p \cdot f$. From a physical viewpoint, the purpose of introducing the notion of a presheaf $\mathbf{P}$ on $\mathcal{B}$, in the environment of the functor category Sets $^{\mathcal{B}^{o p}}$, is to identify an element of $\mathbf{P}(\mathrm{B})$, that is, $p \in \mathbf{P}(\mathrm{B})$, with an event observed by means of a physical procedure over a Boolean domain cover for a quantum event algebra. This identification forces the interrelation of observed events, over all Boolean reference frames of the base category $\mathcal{B}$, to fulfil the requirements of a uniform and homologous fibred structure, further considered in Section 5 .

We notice now that each Boolean algebra $B$ of $\mathcal{B}$ gives naturally rise to a contravariant Hom-functor $\mathbf{y}[B]:=\operatorname{Hom}_{\mathcal{B}}(-, B)$. This functor defines a presheaf on $\mathcal{B}$ for each $B$ in $\mathcal{B}$. Concomitantly, the functor $\mathbf{y}$ is a full and faithful functor from $\mathcal{B}$ to the contravariant functors on $\mathcal{B}$, i.e.,

$$
\mathbf{y}: \mathcal{B} \longrightarrow \operatorname{Sets}^{\mathcal{B}^{o p}}
$$

defining an embedding $\mathcal{B} \hookrightarrow \operatorname{Sets}^{\mathcal{B}^{o p}}$, which is called the Yoneda embedding (Mac Lane and Moerdijk 1992, p. 26; Awodey 2010, pp. 187-189). According to the Yoneda Lemma, there exists an injective correspondence between elements of the set $\mathbf{P}(B)$ and natural transformations in Sets $^{\mathcal{B}^{o p}}$ from $\mathbf{y}[B]$ to $\mathbf{P}$ and this correspondence is natural in both $\mathbf{P}$ and $B$, for every presheaf of sets $\mathbf{P}$ in $\operatorname{Sets}^{\mathcal{B}^{\text {op }}}$ and Boolean algebra $B$ in $\mathcal{B}$. The functor category of presheaves of sets on Boolean event algebras $\mathbf{S e t s}^{\mathcal{B}^{o p}}$ is a complete and co-complete category. Thus, the Yoneda embedding $\mathbf{y}: \mathcal{B} \longrightarrow$ Sets $^{\mathcal{B}^{o p}}$ constitutes the sought free completion of $\mathcal{B}$ under colimits of diagrams of Boolean event algebras.

Then, the Boolean realization functor of a quantum categorical event structure $\mathcal{L}$ in Sets $^{\mathcal{B}^{\text {op }}}$, namely, the functor of generalized elements of $\mathcal{L}$ in the environment of the category of presheaves on Boolean event algebras, is defined as

$$
\mathbf{R}: \mathcal{L} \rightarrow \operatorname{Sets}^{\mathcal{B}^{o p}}
$$

where the action on a Boolean algebra $B$ in $\mathcal{B}$ is given by

$$
\mathbf{R}(L)(B):=\mathbf{R}_{L}(B)=\operatorname{Hom}_{\mathcal{L}}(\mathbf{M}(B), L) .
$$

The functor $\mathbf{R}(L)(-):=\mathbf{R}_{L}(-)=H \operatorname{Hom}_{\mathcal{L}}(\mathbf{M}(-), L)$ is called the functor of Boolean frames of $L$, where $\mathbf{M}: \mathcal{B} \rightarrow \mathcal{L}$ is a Boolean modeling functor of $\mathcal{L}$, i.e., a forgetful functor assigning to each Boolean event algebra the underlying quantum event algebra and to each Boolean homomorphism the underlying quantum algebraic homomorphism. In this manner, a Boolean event algebra $B$, instantiated by the measurement of a quantum observable via the spectral decomposition of the corresponding self-adjoint operator, acts as a partial coordinatizing logical frame of a quantum event algebra $L$ in terms of Boolean coefficients. Notice that the Boolean logical frames of $L$, denoted by

$$
\psi_{B}: \mathbf{M}(B) \rightarrow L
$$

being instantiated by the evaluation of the functor $\mathbf{R}(L)(-)$ at each $B$ in $\mathcal{B}$, are not ad hoc but they are interrelated by the operation of presheaf restriction. Explicitly, this means that for each Boolean homomorphism $f: C \rightarrow B, \mathbf{R}(L)(f): \mathbf{R}(L)(B) \rightarrow \mathbf{R}(L)(C)$ is a function between sets of Boolean frames in the opposite direction such that, if $\psi_{B} \in \mathbf{R}(L)(B)$ is a Boolean frame, the value of $\mathbf{R}(L)(f)\left(\psi_{B}\right)$, or equivalently, the corresponding Boolean 
frame $\psi_{C}: \mathbf{M}(C) \rightarrow L$ is given by the restriction or pullback of $\psi_{B}$ along $f$, denoted by $\mathbf{R}(L)(f)\left(\psi_{B}\right)=\psi_{B} \cdot f=\psi_{C}$.

In this setting, the problem of establishing a functorial representation of quantum event algebras is solved exactly by finding the left adjoint functor $\mathbf{L}: \operatorname{Sets}^{\mathcal{B}^{o p}} \rightarrow \mathcal{L}$ to the Boolean realization functor $\mathbf{R}: \mathcal{L} \rightarrow \operatorname{Sets}^{\mathcal{B}^{\text {op }}}$. In other words, the existence of the left adjoint functor $\mathbf{L}$ paves the way for an explicit reconstruction of quantum event algebras by means of appropriate diagrams of Boolean logical frames in a structure-preserving manner. Additionally, as will be shown in the sequel, it can be used for addressing efficiently the problem of truth valuation in the quantum domain by circumventing the Kochen-Specker types of prohibition. This becomes possible by exploiting the topos logical structure of the category of diagrams of Boolean frames, and then using the left adjoint functor for the classification of quantum event algebras in terms of contextual truth valuations with respect to interconnected covering families of local Boolean frames.

In this context of reasoning, we can show that there exists a categorical adjunction between the categories Sets $^{\mathcal{B}^{o p}}$ and $\mathcal{L}$. More precisely, there exists a pair of adjoint functors $\mathbf{L} \dashv \mathbf{R}$ as follows (Zafiris 2004):

$$
\mathbf{L}: \operatorname{Sets}^{\mathcal{B}^{o p}} \longleftarrow \mathcal{L}: \mathbf{R} .
$$

The Boolean frames-quantum adjunction consists of the functors $\mathbf{L}$ and $\mathbf{R}$, called left and right adjoints, as well as the natural bijection:

$$
\operatorname{Nat}(\mathbf{P}, \mathbf{R}(L)) \cong \operatorname{Hom}_{\mathcal{L}}(\mathbf{L P}, L) .
$$

The established bijective correspondence, interpreted functorially, assures that the Boolean realization functor of $\mathcal{L}$, realized for each $L$ in $\mathcal{L}$ by its functor of Boolean frames,

$$
\mathbf{R}(L): B \mapsto \operatorname{Hom}_{\mathcal{L}}(\mathbf{M}(B), L),
$$

has a left adjoint functor $\mathbf{L}:$ Sets $^{\mathcal{B}^{o p}} \rightarrow \mathcal{L}$, which is defined for each presheaf $\mathbf{P}$ of Boolean algebras in Sets $^{\mathcal{B}}{ }^{\mathcal{P}}$ as the colimit $\mathbf{L}(\mathbf{P})$, explicitly constructed for the case of interest $\mathbf{P}=$ $\mathbf{R}(L)$ in the next section. Thus, the following diagram, where the Yoneda embedding is denoted by $\mathbf{y}$, commutes:

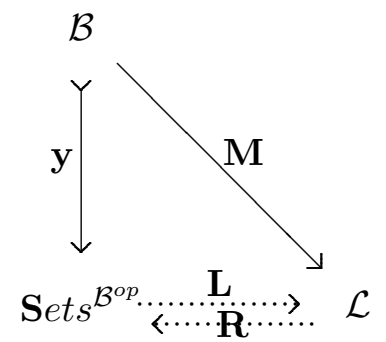

The pair of adjoint functors $\mathbf{L} \dashv \mathbf{R}$ formalizes categorically the process of encoding and decoding information between diagrams of Boolean frames and quantum event algebras respecting their distinctive structural form. In general, the existence of an adjunction between two categories always gives rise to a family of universal morphisms, called unit and counit of the adjunction, one for each object in the first category and one for each object in the second. In this way, each object in the first category induces a certain property in the second category and the universal morphism carries the object to the universal for that property. Most importantly, every adjunction extends to an adjoint equivalence of certain subcategories of the initial functorially correlated categories. It is precisely this category-theoretic fact which determines the necessary and sufficient conditions for the isomorphic representation of quantum event algebras by means of suitably restricted functors of Boolean frames.

Every categorical adjunction is completely characterized by the unit and counit natural transformations, acquiring the status of universal mapping properties (Awodey 2010, pp. 208215). In relation to the Boolean frames-quantum adjunction, for any presheaf $\mathbf{P}$ of Boolean 
event algebras in the functor category Sets $^{B^{o p}}$, the unit of the adjunction is defined as

$$
\delta_{\mathbf{P}}: \mathbf{P} \longrightarrow \mathbf{R L P} .
$$

On the other side, for each quantum event algebra $L$ in $\mathcal{L}$, the counit is defined as

$$
\epsilon_{L}: \mathbf{L R}(L) \longrightarrow L
$$

The representation of a quantum event algebra $L$ in $\mathcal{L}$, in terms of the functor of Boolean frames $\mathbf{R}(L)$ of $L$, is full and faithful if and only if the counit of the Boolean frames-quantum adjunction is a quantum algebraic isomorphism, that is structure-preserving, injective and surjective. In turn, the counit of the Boolean frames-quantum adjunction is a quantum algebraic isomorphism if and only if the right adjoint functor is full and faithful. In the latter case we characterize the Boolean modeling functor $\mathbf{M}: \mathcal{B} \rightarrow \mathcal{L}$ as a proper or dense modeling functor.

\section{Equivalence Classes of Pointed Boolean Frames}

The functorial representation of a quantum event algebra $L$ in $\mathcal{L}$ through the category of presheaves of Boolean event algebras Sets $^{\mathcal{B}^{o p}}$ requires an explicit calculation of the colimit, $\mathbf{L}(\mathbf{P})=\mathbf{L R}(L)$, when the functor on which it acts is the presheaf functor of Boolean frames of $L$. This task is simplified by the observation that there exists an underlying colimit-preserving faithful functor from the category $\mathcal{L}$ to the category Sets. Thus, we can calculate the colimit in Sets by means of equivalence classes and, then, show how the obtained set of equivalence classes carries the structure of a quantum event algebra.

In order to calculate, in general, the colimit $\mathbf{L}(\mathbf{P})$ for any $\mathbf{P}$ in $\mathbf{S e t s}^{\mathcal{B}^{o p}}$, it is necessary to specify the index or parameterizing category corresponding to the functor $\mathbf{P}$, which is defined over the base category of Boolean event algebras $\mathcal{B}$. This index category is called the category of elements of a presheaf $\mathbf{P}$, denoted by $\int(\mathbf{P}, \mathcal{B})$, and defined as follows: it has objects all pairs $(B, p)$ and morphisms $(\dot{B}, \dot{p}) \rightarrow(B, p)$ are those morphisms $u: \dot{B} \rightarrow B$ of $\mathcal{B}$ for which $p \cdot u=\dot{p}$, that is the restriction or pullback of $p$ along $u$ is $p$. Projection on the second coordinate of $\int(\mathbf{P}, \mathcal{B})$ defines a functor $\int_{\mathbf{P}}: \int(\mathbf{P}, \mathcal{B}) \rightarrow \mathcal{B}$ called the split discrete fibration induced by $\mathbf{P}$, where $\mathcal{B}$ is the base category of the fibration, as in the diagram below. Notice that the fibers of this fibration are categories in which the only arrows are identity arrows, i.e., they are actually sets. Then, if $B$ is an object of $\mathcal{B}$, the inverse image under $\int_{\mathbf{P}}$ of $B$ is simply the set $\mathbf{P}(B)$, although its elements are written as pairs so as to form a disjoint union.

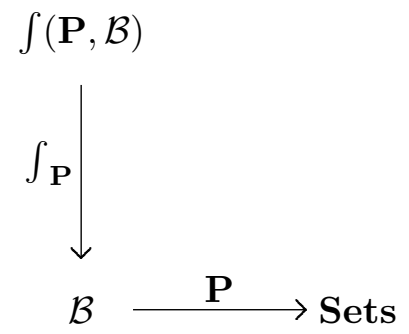

Consequently, the index category corresponding to the functor $\mathbf{P}$ is the category of its elements $\mathcal{I} \equiv \int(\mathbf{P}, \mathcal{B})$, whence the functor $\left[\mathbf{M} \circ \int_{\mathbf{P}}\right]$ defines the diagram $\mathbf{X}: \mathcal{I} \rightarrow \mathcal{L}$ over which the colimit should be calculated. Hence, we obtain:

$$
\mathbf{L}(\mathbf{P})=\mathbf{L}_{\mathbf{M}}(\mathbf{P})=\operatorname{Colim}\left\{\int(\mathbf{P}, \mathcal{B}) \stackrel{\int_{\mathbf{P}}}{\longrightarrow} \mathcal{B} \stackrel{\mathbf{M}}{\longrightarrow} \mathcal{L} \rightarrow \mathbf{\text { Sets }}\right\}
$$

Now we consider the case of our interest where $\mathbf{P}=\mathbf{R}(L)$, i.e., the presheaf functor $\mathbf{P}$ represents the functor of Boolean frames of a quantum event algebra $L$. In this case, the 
category of elements $\int(\mathbf{R}(\mathbf{L}), \mathcal{B})$ has objects all pairs $\left(B, \psi_{B}\right)$, where $B$ is a Boolean event algebra and $\psi_{B}: \mathbf{M}(B) \rightarrow L$ is a Boolean frame of $L$ defined over $B$. The morphisms of $\int(\mathbf{R}(\mathbf{L}), \mathcal{B})$, denoted by $\left(\dot{B}, \psi_{\dot{B}}\right) \rightarrow\left(B, \psi_{B}\right)$, are those Boolean event algebra homorphisms $u: B \rightarrow B$ of the base category $\mathcal{B}$ for which $\psi_{B} \cdot u=\psi_{\hat{B}}$, that is the restriction or pullback of the Boolean frame $\psi_{B}$ along $u$ is $\psi_{\dot{B}}$.

Next, we define the set of pointed Boolean frames of a quantum event algebra $L$ as

$$
\mathbf{Y}\left(\mathbf{R}_{L}\right)=\left\{\left(\psi_{B}, q\right) /\left(\psi_{B}: \mathbf{M}(B) \rightarrow L\right), q \in \mathbf{M}(B)\right\} .
$$

Note that a pointed Boolean frame $\zeta:=\left(\psi_{B}, q\right)$ of $L$ over $B$ consists of a Boolean frame $\psi_{B}: \mathbf{M}(B) \rightarrow L$ together with a projection operator $q \in \mathbf{M}(B)$. The crucial observation is that the composition law in the category $\int(\mathbf{R}(\mathbf{L}), \mathcal{B})$ induces a transitive and reflexive relation $\Re$ on the set $\mathbf{Y}\left(\mathbf{R}_{L}\right)$, defined as follows,

$$
\left(\psi_{B} \cdot u, \dot{q}\right) \Re\left(\psi_{B}, u(\dot{q})\right),
$$

for any Boolean homomorphism $u: B \rightarrow B$ in the base category $\mathcal{B}$. The next step is to make this relation also symmetric by postulating that for pointed Boolean frames $\zeta, \eta$ in $\mathbf{Y}\left(\mathbf{R}_{L}\right)$, where $\zeta, \eta$ denote pairs in the above set, we have

$$
\zeta \sim \eta
$$

if and only if $\zeta \Re \eta$ or $\eta \Re \zeta$. Finally, by considering a sequence $\xi_{1}, \xi_{2}, \ldots, \xi_{k}$ of pointed Boolean frames in the set $\mathbf{Y}\left(\mathbf{R}_{L}\right)$ and also $\zeta, \eta$ such that

$$
\zeta \sim \xi_{1} \sim \xi_{2} \sim \ldots \sim \xi_{k-1} \sim \xi_{k} \sim \eta
$$

we define an equivalence relation on the set $\mathbf{Y}\left(\mathbf{R}_{L}\right)$ as follows,

$$
\zeta \bowtie \eta:=\zeta \sim \xi_{1} \sim \xi_{2} \sim \ldots \sim \xi_{k-1} \sim \xi_{k} \sim \eta
$$

if and only if there exists a path of Boolean transition homomorphisms in $\mathcal{B}$ inducing the above equivalence. Then, for each pair $\zeta=\left(\psi_{B}, q\right) \in \mathbf{Y}\left(\mathbf{R}_{L}\right)$, we define the equivalence class at pointed Boolean frame $\zeta$ :

$$
Q_{\zeta}=\left\{\iota \in \mathbf{Y}\left(\mathbf{R}_{L}\right): \zeta \bowtie \iota\right\}
$$

We finally define the quotient set of equivalence classes of pointed Boolean frames as follows,

$$
\mathbf{Y}\left(\mathbf{R}_{L}\right) / \bowtie:=\left\{Q_{\zeta}: \zeta=\left(\psi_{B}, q\right) \in \mathbf{Y}\left(\mathbf{R}_{L}\right)\right\},
$$

and use the notation $Q_{\zeta}=\left\|\left(\psi_{B}, q\right)\right\|=\psi_{B} \otimes q$ to denote the equivalence class at a single pointed Boolean frame $\zeta=\left(\psi_{B}, q\right)$. The quotient set $\mathbf{Y}\left(\mathbf{R}_{L}\right) / \bowtie$ completes the calculation of the colimit $\mathbf{L R}(L)$ in Sets. The tensor notation is justified by the fact that the equivalence classes of the form $\psi_{B} \otimes q$ are canonically identified as elements of the tensor product of the functors $\mathbf{R}(L)$ and $\mathbf{M}$ over the base category $\mathcal{B}$. Thus, we finally obtain:

$$
\mathbf{L R}(L) \cong\left\{\mathbf{Y}\left(\mathbf{R}_{L}\right) / \bowtie\right\} \equiv \mathbf{R}(L) \otimes_{\mathcal{B}} \mathbf{M}
$$

Importantly, the quotient set $\mathbf{R}(L) \otimes_{\mathcal{B}} \mathbf{M}$ is naturally endowed with a quantum event algebraic structure as follows. First, the orthocomplementation is defined by the assignment:

$$
Q_{\zeta}^{*}=\left(\psi_{B} \otimes q\right)^{*}:=\psi_{B} \otimes q^{*} .
$$

Second, the unit element is defined by:

$$
1:=\psi_{B} \otimes 1
$$


Third, two equivalence classes in the quotient set $\mathbf{R}(L) \otimes_{\mathcal{B}} \mathbf{M}$ can be ordered if and only if they have a common refinement. Consequently, the partial order structure is defined by the assignment,

$$
\left(\psi_{B} \otimes q\right) \preceq\left(\psi_{C} \otimes r\right),
$$

if and only if,

$$
d_{1} \preceq d_{2},
$$

where we have made the following identifications,

$$
\begin{aligned}
& \left(\psi_{B} \otimes q\right)=\left(\psi_{D} \otimes d_{1}\right) \\
& \left(\psi_{C} \otimes r\right)=\left(\psi_{D} \otimes d_{2}\right),
\end{aligned}
$$

with $d_{1}, d_{2} \in \mathbf{M}(D)$, according to the pullback diagram,

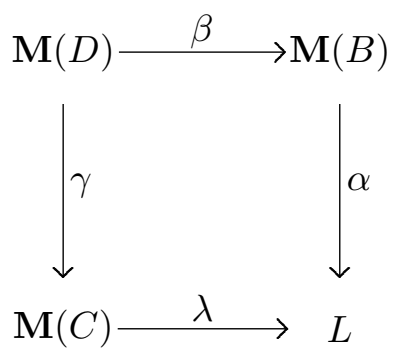

such that $\beta\left(d_{1}\right)=q, \gamma\left(d_{2}\right)=r$, and $\beta: \mathbf{M}(D) \rightarrow \mathbf{M}(B), \gamma: \mathbf{M}(D) \rightarrow \mathbf{M}(C)$ denote the pullback of $\alpha: \mathbf{M}(B) \rightarrow L$ along $\lambda: \mathbf{M}(C) \rightarrow L$ in the category of quantum event algebras.

We conclude that the ordering relation between any two equivalence classes of pointed Boolean frames in the set $\mathbf{R}(L) \otimes_{\mathcal{B}} \mathbf{M}$ requires the existence of pullback compatibility conditions between the corresponding Boolean frames, established in the next section. This is a consideration of central significance for an isomorphic representation of quantum event algebras in terms of pullback compatible Boolean frames.

\section{Boolean Localization Functors}

In confronting this issue, let us recall that for each quantum event algebra $L$ in $\mathcal{L}$ the counit of the Boolean frames-quantum adjunction is defined as

$$
\epsilon_{L}: \mathbf{L R}(L) \longrightarrow L \text {. }
$$

If we express the calculation of the colimit in terms of equivalence classes of pointed Boolean frames, viz. $\mathbf{L R}(L) \cong \mathbf{R}(L) \otimes_{\mathcal{B}} \mathbf{M}$, we obtain:

$$
\epsilon_{L}: \mathbf{R}(L) \otimes_{\mathcal{B}} \mathbf{M} \longrightarrow L .
$$

Thus, the counit $\epsilon_{L}$ fits into the following diagram:

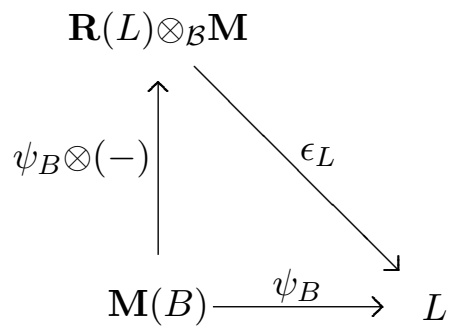


Accordingly, for every Boolean frame $\psi_{B}: \mathbf{M}(B) \rightarrow L$ the projection operator $q \in \mathbf{M}(B)$ is mapped to an event in $L$ only through its factorization via the equivalence class $\psi_{B} \otimes q$ of pointed Boolean frames, or equivalently,

$$
\epsilon_{L}\left(\left[\psi_{B} \otimes q\right]\right)=\psi_{B}(q), \quad q \in \mathbf{M}(B) .
$$

We note that if the counit natural transformation $\epsilon_{L}$ at $L$ can be appropriately restricted to an isomorphism $\mathbf{R}(L) \otimes_{\mathcal{B}} \mathbf{M} \cong L$, then the structural information of $L$ can be completely encoded and classified logically through equivalence classes of pointed Boolean frames. For this purpose, we need to impose suitable conditions on families of Boolean frames in $\mathbf{R}(L)$, which are going to play the role of local Boolean covers of $L$. Intuitively, we require that local Boolean covers of $L$ should constitute a minimal generating class of Boolean frames which jointly should form an epimorphic family covering $L$ entirely on their overlaps, and moreover they should be compatible under refinement operations. We formulate these notions as follows.

A functor of Boolean coverings for a quantum event algebra $L$ in $\mathcal{L}$ is defined as a subfunctor $\mathbf{T}$ of the functor of Boolean frames $\mathbf{R}(L)$ of $L$,

$$
\mathbf{T} \hookrightarrow \mathbf{R}(L) .
$$

For each Boolean algebra $B$ in $\mathcal{B}$, a subfunctor $\mathbf{T} \hookrightarrow \mathbf{R}(L)$ is equivalent to an algebraic right ideal or sieve of quantum homomorphisms $\mathbf{T} \triangleright \mathbf{R}(L)$, defined by the requirement that, for each $B$ in $\mathcal{B}$, the set of elements of $\mathbf{T}(B) \subseteq[\mathbf{R}(L)](B)$ is a set of Boolean frames $\psi_{B}: \mathbf{M}(B) \rightarrow L$ of $\mathbf{R}(L)(B)$, called Boolean covers of $L$, satisfying the following property:

\{If $\left[\psi_{B}: \mathbf{M}(B) \rightarrow L\right] \in \mathbf{T}(B)$, viz. it is a Boolean cover of $L$, and $\mathbf{M}(v): \mathbf{M}(B) \rightarrow \mathbf{M}(B)$ in $\mathcal{L}$ for $v: B \rightarrow B$ in $\mathcal{B}$, then $\left[\psi_{B} \circ \mathbf{M}(v): \mathbf{M}(\dot{B}) \rightarrow \mathcal{L}\right] \in \mathbf{T}(B)$, viz. it is also a Boolean cover of $L\}$.

A family of Boolean covers $\psi_{B}: \mathbf{M}(B) \longrightarrow L, B$ in $\mathcal{B}$, is the generator of an ideal of Boolean coverings $\mathbf{T}$, if and only if, this ideal is the smallest among all containing that family. The ideals of Boolean coverings for an $L$ in $\mathcal{L}$ constitute a partially ordered set under inclusion of subobjects. The minimal ideal is the empty one, namely $\mathbf{T}(B)=\emptyset$ for all $B$ in $\mathcal{B}$, whereas the maximal ideal is the set of all Boolean frames of $L$ for all $B$ in $\mathcal{B}$.

We remind that the ordering relation between any two equivalence classes of pointed Boolean frames in the set $\mathbf{R}(L) \otimes_{\mathcal{B}} \mathbf{M}$ requires the existence of pullback compatibility between the corresponding Boolean frames. Thus, if we consider a functor of Boolean coverings $\mathbf{T}$ for a quantum event algebra $L$, we require that the generating family of Boolean covers they belong to is compatible under pullbacks. Then, the pairwise gluing isomorphism of the Boolean covers $\psi_{B}: \mathbf{M}(B) \longrightarrow L, B$ in $\mathcal{B}$, and $\psi_{\dot{B}}: \mathbf{M}(\dot{B}) \longrightarrow L, \dot{B}$ in $\mathcal{B}$, is defined as

$$
\begin{gathered}
\Omega_{B, \dot{B}}: \psi_{\dot{B} B}\left(\mathbf{M}(B) \times{ }_{L} \mathbf{M}(\dot{B})\right) \longrightarrow \psi_{B \dot{B}}\left(\mathbf{M}(B) \times{ }_{L} \mathbf{M}(\dot{B})\right) \\
\Omega_{B, \dot{B}}=\psi_{B \dot{B}} \circ \psi_{\dot{B} B}{ }^{-1},
\end{gathered}
$$

where $\mathbf{M}(B) \times{ }_{L} \mathbf{M}(\dot{B})$, together with the two projections $\psi_{B \dot{B}}$ and $\psi_{\dot{B} B}$, is the pullback or categorical overlap of the Boolean covers $\psi_{B}: \mathbf{M}(B) \longrightarrow L, B$ in $\mathcal{B}$, and $\psi_{\dot{B}}: \mathbf{M}(\dot{B}) \longrightarrow L, \dot{B}$ in $\mathcal{B}$, with common codomain a quantum event algebra $L$, as shown in the diagram:

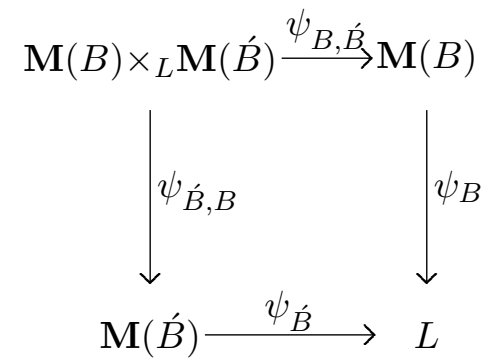


An immediate consequence of the previous definition is the satisfaction of the following Boolean coordinate cocycle conditions for injective Boolean covers:

$$
\begin{gathered}
\Omega_{B, B}=1_{B} \quad 1_{B} \text { : identity of } B \\
\Omega_{B, \dot{B}} \circ \Omega_{\dot{B}, \dot{B}}=\Omega_{B, \dot{B}} \\
\Omega_{B, \dot{B}}=\Omega_{B, B}^{-1} \quad \text { if } \mathbf{M}(B) \cap \mathbf{M}(\dot{B}) \cap \mathbf{M}(\dot{B}) \neq 0
\end{gathered}
$$

Thus, the gluing isomorphism assures that the Boolean covers $\psi_{\dot{B} B}:\left(\mathbf{M}(B) \times{ }_{L} \mathbf{M}(\dot{B})\right) \rightarrow L$ and $\psi_{B \dot{B}}:\left(\mathbf{M}(B) \times{ }_{L} \mathbf{M}(\dot{B})\right) \rightarrow L$ cover the same part of $L$ compatibly.

Now, given a functor of Boolean coverings $\mathbf{T}$ for a quantum event algebra $L$, we call it a functor of Boolean localizations of $L$, or a structure sheaf of Boolean coefficients of $L$, if and only if the Boolean coordinate cocycle conditions are satisfied. Then, we can show that for a dense generating family of Boolean covers in a Boolean localization functor $\mathbf{T}$ of $L$, meaning that they generate an epimorphic ideal of Boolean coverings of $L$, the counit of the Boolean frames-quantum adjunction is restricted to a quantum algebraic isomorphism, that is structure-preserving, injective and surjective (Zafiris 2004). In turn, the right adjoint functor of the adjunction restricted to a Boolean localization functor is full and faithful. This argument can be formalized more precisely in topos theoretic terminology using the technical means of a subcanonical Grothendieck topology (called the topology of epimorphic families) on the base category of Boolean algebras. For details the interested reader should consult (Zafiris 2006b). Consequently, $\mathcal{L}$ is a reflection of the topos of variable local Boolean frames Sets $^{B^{o p}}$, and the structure of a quantum event algebra $L$ in $\mathcal{L}$ is preserved by the action of a family of Boolean frames if and only if this family forms a Boolean localization functor of $L$. The next step is to show that the category of quantum event algebras $\mathcal{L}$ is equipped with a subobject classifier or classifying object acting as an object of truth values for the valuation of propositions describing the behavior of quantum systems.

\section{Existence of the Subobject Classifier in $\mathcal{L}$}

A proper understanding of the meaning of the existence of a subobject classifier in the category of quantum event algebras presupposes the clarification of the notion of subobjects in any categorical environment, since it is going to be the main conceptual tool in our argumentation. A subobject of an object $X$ in any category $\mathcal{X}$ with pullbacks is an equivalence class of monic arrows targeting $X$, denoted by $\mu: M \hookrightarrow X$. The set of all subobjects of $X$ in the category $\mathcal{X}$, denoted by $\Theta_{\mathcal{X}}(X)$, is a partially ordered set under inclusion of subobjects. Now, $\boldsymbol{\Theta}_{\mathcal{X}}$ can be construed as a presheaf functor in Sets ${ }^{\mathcal{X} p}$ by the operation of pulling back as follows. Given an arrow $g: Y \rightarrow X$ in $\mathcal{X}$, the pullback of any monic arrow $\mu: M \hookrightarrow X$ along the arrow $g$ is a new monic arrow $\dot{\mu}: M^{\prime} \hookrightarrow Y$, that is a subobject of $Y$, and obviously the assignment $\mu \mapsto \mu ́$ defines a function $\boldsymbol{\Theta}_{\mathcal{X}}(g): \boldsymbol{\Theta}_{\mathcal{X}}(X) \rightarrow \boldsymbol{\Theta}_{\mathcal{X}}(Y)$.

An immediate question that arises in this setting is related with the possibility of representing the subobject functor $\boldsymbol{\Theta}_{\mathcal{X}}$ in Sets ${ }^{\mathcal{X}^{o p}}$ internally by an object $\Omega$ in the category $\mathcal{X}$ such that, for each $X$ in $\mathcal{X}$, there exists a natural isomorphism:

$$
\iota_{X}: \Theta_{\mathcal{X}}(X) \cong \operatorname{Hom}_{\mathcal{X}}(X, \Omega)
$$

If the subobject functor becomes representable with representing object $\Omega$ in $\mathcal{X}$, then we say that the category $\mathcal{X}$ is equipped with a subobject classifier. By this term we mean a universal monic arrow

$$
T:=\text { True }: 1 \hookrightarrow \Omega
$$

such that, to every monic arrow $\mu: M \hookrightarrow X$ in $\mathcal{X}$, there is a unique characteristic arrow $\phi_{\mu}$, which, with the given monic arrow $\mu$, forms a pullback diagram in $\mathcal{X}$ : 


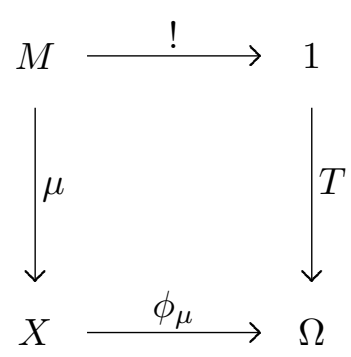

This is equivalent to saying that every subobject of $X$ in $\mathcal{X}$ is uniquely a pullback of the universal monic $T$. Conversely, satisfaction of this property amounts to saying that the subobject functor $\boldsymbol{\Theta}_{\mathcal{X}}$ is representable by the object $\Omega$, or equivalently, that it is isomorphic to $\operatorname{Hom}_{\mathcal{X}}(-, \Omega)$. Notice that the bijection $\iota_{X}$ sends each subobject $\mu: M \hookrightarrow X$ of $X$ to its unique characteristic arrow $\phi_{\mu}: X \rightarrow \Omega$, and conversely.

A particularly important example, where the subobject classifier of a category always exists, is the case when the category $\mathcal{X}$ is a topos. The paradigmatic instance of the function of the subobject classifier in a topos is provided by the classifying function of the two-valued object $2:=\{$ false,true $\}=\{0,1\}$ in the topos Sets of normal sets. The terminal object in Sets is given by $1=\{*\}$, i.e., by the one-point set. A subobject $\mu: M \hookrightarrow X$ of a set $X$ is just a subset $M \subset X$, which is classified by the characteristic function $\phi_{\mu}: X \rightarrow 2$ on the set $X$, defined as follows: for every $x \in X, \phi_{\mu}(x)=1=$ true if $x \in M$, and $\phi_{\mu}(x)=0=$ false otherwise. Then, by using the injective map True : $1=\{*\} \rightarrow\{$ false,true $\}=\{0,1\}$ defined by $\{*\} \mapsto 1$, we indeed obtain the subobject classifier in Sets, where $\Omega=2$ classifies subsets of $X$ in Sets according to the pullback diagram:

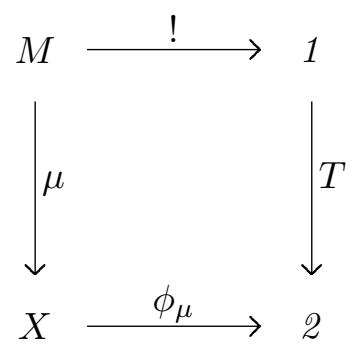

Thus, in the topos Sets the two-valued Boolean algebra $2=\{$ false,true $\}=\{0,1\}$ is the classifying object or object of truth values, which means that the internal logic of the category Sets is the usual interpretation of classical logic. Equivalently, we may say that the category of Boolean event algebras $\mathcal{B}$ is classified internally by the two-valued Boolean event algebra 2.

In contradistinction with the preceding case, as already noted in Section 2, KochenSpecker's theorem prohibits the existence of a global classifying two-valued homomorphism on a quantum event algebra, and thus, the latter cannot be embedded into a Boolean event algebra. Due to this, a pertinent issue regarding the logic of quantum events is to find out if there exists another classifying object $\Omega$ in $\mathcal{L}$ acting as an object of truth values in analogy with the classical case. The representation of quantum event algebras in terms of Boolean localization functors is particularly suited to address this issue. It is useful to recapitulate the role of Boolean localization functors by means of the following diagram displaying the function of the counit of the Boolean frames-quantum adjunction:

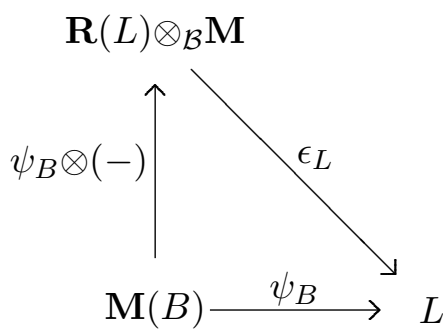


If the functor of Boolean frames $\mathbf{R}(L)$ of $L$ is restricted to a Boolean localization functor $\mathbf{T}$, then the counit of the adjunction localized to $\mathbf{T}$ becomes an isomorphism, and thus quantum events are represented isomorphically in terms of equivalence classes $\psi_{B} \otimes q, q \in \mathbf{M}(B)$, of pointed Boolean covers, namely pointed Boolean frames qualified as local covers of $L$. Thus, the action of Boolean localization functors on quantum event algebras $L$ makes the category $\mathcal{L}$ a reflection of Sets $^{B^{o p}}$. Technically speaking, this means that $\mathcal{L}$ is a complete category and monic arrows are preserved by the right adjoint Boolean realization functor of quantum event algebras. In particular, there exist a terminal object and pullbacks of monic arrows (Mac Lane and Moerdijk 1992). Thus, there exists a subobject functor for a quantum categorical event structure $\mathcal{L}$ equipped with Boolean localization functors.

The subobject functor of $\mathcal{L}$ is defined as follows:

$$
\text { Sub : } \mathcal{L}^{o p} \rightarrow \text { Sets. }
$$

The functor Sub is a contravariant functor by pulling back. Composition with a proper or dense Boolean modeling functor defines a presheaf in Sets ${ }^{B^{o p}}$, called the Boolean frames modeled subobject functor of $\mathcal{L}$, as follows:

$$
\text { Sub } \circ \mathbf{M}: \mathcal{B}^{o p} \rightarrow \mathcal{L}^{o p} \rightarrow \text { Sets. }
$$

In a compact notation we obtain,

$$
\Upsilon_{\mathbf{M}}:=\Upsilon(\mathbf{M}(-)):=\mathbf{S u b} \circ \mathbf{M}: \mathcal{B}^{o p} \rightarrow \mathbf{S e t s},
$$

such that,

$$
\mathcal{B}^{o p} \ni B \mapsto\{[\operatorname{Dom}(m) \hookrightarrow \mathbf{M}(B)]\} \in \mathbf{S e t s},
$$

where the range denotes the set of subobjects of $\mathbf{M}(B)$, i.e., the set of equivalence classes of monic quantum homomorphisms $m$ from $\operatorname{Dom}(m)$ to $\mathbf{M}(B)$.

The set $\Upsilon_{\mathbf{M}}(B)=\Upsilon(\mathbf{M}(B))$ is defined as the set of all subobjects of $\mathbf{M}(B)$, for every $B$ in $\mathcal{B}$, in the category $\mathcal{L}$. Notice that the set $\Upsilon(\mathbf{M}(B))$, for every $B$ in $\mathcal{B}$, is a partially ordered set under inclusion of subobjects of $\mathbf{M}(B)$.

We emphasize that $\Upsilon_{\mathbf{M}}$ is an object in Sets ${ }^{B^{o p}}$, so the pertinent question is if it can be represented internally in $\mathcal{L}$ by means of a concrete quantum event algebra $\Omega$, which would play in this manner the role of a truth-values object, or equivalently, a subobject classifier in $\mathcal{L}$, defined as follows.

The subobject classifier of the category of quantum event algebras is a universal monic quantum homomorphism

$$
T:=\text { True }: 1 \hookrightarrow \Omega
$$

such that, for every subobject $K$ of $L$ in $\mathcal{L}$, represented by the monic arrow $m: K \hookrightarrow L$, there is a unique characteristic arrow $\phi_{m}$, which, with the given monic arrow $m$, forms a pullback diagram:

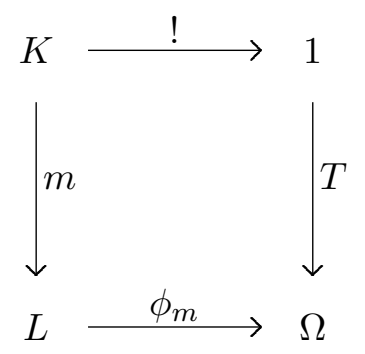

This is equivalent to saying that every subobject of $L$ in $\mathcal{L}$ is uniquely a pullback of the universal monic $T$. 
The significance of the internal representation of $\Upsilon_{M}$ in $\mathcal{L}$ boils down to the fact that if this is the case, we could legitimately interpret the concrete classifying object $\Omega$ as a truthvalues object in $\mathcal{L}$, and consequently use it to classify quantum propositions circumventing in this way the Kochen-Specker prohibition. Thus, in this case, subobjects of a quantum event algebra should be characterized in terms of characteristic functions, which take values not in 2 , but precisely in the truth-values object $\Omega$ in $\mathcal{L}$. Most important, in that case the category of quantum event algebras $\mathcal{L}$ would be endowed with a subobject classifier.

\subsection{The quantum object of truth values}

The issue of the existence of a classifying or truth-values object $\Omega$ in $\mathcal{L}$ can be fruitfully examined from the perspective of the Boolean-frames quantum adjunction. We have seen previously that the counit of the adjunction, $\epsilon_{L}: \mathbf{L R}(L) \longrightarrow L$, evaluated at any quantum event algebra $L$ in $\mathcal{L}$, when restricted to a Boolean localization functor of $L$ becomes an isomorphism. In particular, if $\Omega$ exists in $\mathcal{L}$, there must be a Boolean localization functor $\mathbf{T}$ of $\Omega$ such that,

$$
\epsilon_{\Omega}: \mathbf{R}(\Omega) \otimes_{\mathcal{B}} \mathbf{M} \cong \Omega,
$$

where $\mathbf{R}(\Omega)$ is localized to $\mathbf{T} \hookrightarrow \mathbf{R}(\Omega)$.

Henceforth, it is easily deduced that the subobject functor $\Upsilon_{\mathbf{M}}$ is representable internally in $\mathcal{L}$ by a classifying or truth-values object $\Omega$ if and only if there exists a natural isomorphism:

$$
\Upsilon(\mathbf{M}(-)) \cong \mathbf{R}(\Omega):=\operatorname{Hom}_{\mathcal{L}}(\mathbf{M}(-), \Omega) .
$$

The naturality condition means that the isomorphism holds at each Boolean algebra $B$ in $\mathcal{B}$.

At the other side of the bidirectional adjunction, we have seen that for any presheaf $\mathbf{P} \in \mathbf{S e t s}^{B^{o p}}$, the unit is defined as $\delta_{\mathbf{P}}: \mathbf{P} \longrightarrow \mathbf{R L P}$. Thus, if we consider as $\mathbf{P} \in \mathbf{S e t s}^{B^{o p}}$ the subobject functor $\Upsilon(\mathbf{M}(-))$, we obtain the following natural transformation

$$
\delta_{\Upsilon(\mathbf{M}(-))}: \Upsilon(\mathbf{M}(-)) \longrightarrow \mathbf{R L} \Upsilon(\mathbf{M}(-))
$$

or equivalently,

$$
\delta_{\Upsilon(\mathbf{M}(-))}: \Upsilon(\mathbf{M}(-)) \longrightarrow \operatorname{Hom}_{\mathcal{L}}(\mathbf{M}(-), \mathbf{L} \Upsilon(\mathbf{M}(-))
$$

Hence, by inspecting the unit $\delta_{\Upsilon(\mathbf{M}(-))}$ evaluated at $\Upsilon(\mathbf{M}(-))$ with respect to a Boolean localization functor of $L$, we arrive at the following conclusion. If the unit $\delta_{\Upsilon(\mathbf{M}(-))}$, restricted to a Boolean localization functor $\mathbf{T}$ of $L$ for every $L$ in $\mathcal{L}$, is an isomorphism, then the subobject functor $\Upsilon(\mathbf{M}(-))$ becomes representable in $\mathcal{L}$ by the quantum truth-values object $\Omega$, defined as follows,

$$
\Omega:=\mathbf{L} \Upsilon(\mathbf{M}(-)),
$$

and thus the category of quantum event algebras is endowed with a subobject classifier.

It is remarkable that the unit of the Boolean fames-quantum adjunction $\delta_{\Upsilon(\mathbf{M}(-))}$, localized at $\mathbf{T}$, depicts exactly the object of truth values $\Omega$ in $\mathcal{L}$, which is represented, in virtue of the counit isomorphism, as the colimit $\Omega:=\mathbf{L} \Upsilon(\mathbf{M}(-))$ in the category of elements of the subobject functor $\Upsilon(\mathbf{M}(-))$. It is straightforward to verify the latter remark, in case the unit $\delta_{\Upsilon(\mathbf{M}(-))}$ is an isomorphism, by noticing that

$$
\Omega:=\mathbf{L} \Upsilon(\mathbf{M}(-)) \cong \mathbf{L}[\mathbf{R L} \Upsilon(\mathbf{M}(-))] \cong \mathbf{L R} \Omega
$$

is precisely an expression of the counit isomorphism for the quantum event algebra $\Omega$ restricted to $\mathbf{T}$.

We emphasize that the above unit isomorphism $\delta_{\Upsilon(\mathbf{M}(-))}$, evaluated at each Boolean algebra $B$ serving as the domain of a local Boolean cover of $\Omega$, means that there exists a bijection 
$\delta_{\Upsilon(\mathbf{M}(B))}:=\Delta$ sending each subobject $\lambda: \operatorname{Dom}(\lambda) \hookrightarrow \mathbf{M}(B)$ of $\mathbf{M}(B)$ to its unique characteristic arrow $\Delta^{\lambda}{ }_{B}: \mathbf{M}(B) \rightarrow \Omega$, and conversely. This is equivalent to saying that every subobject of $\mathbf{M}(B)$ in $\mathcal{L}$ is uniquely a pullback of the universal monic $T$. Thus, the following diagram is a classifying pullback diagram in $\mathcal{L}$ for each quantum algebraic homomorphism or classifying arrow

$$
\Delta^{\lambda}{ }_{B}: \mathbf{M}(B) \rightarrow \Omega
$$

from the domain of a Boolean cover $\mathbf{M}(B)$, such that $\lambda$ is a subobject of $\mathbf{M}(B)$ :

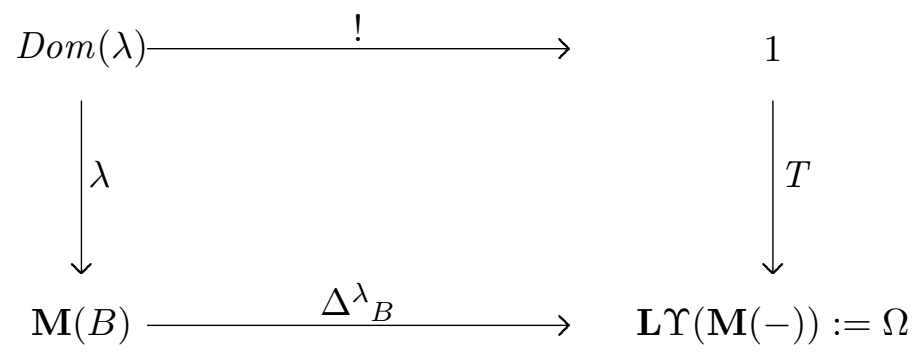

Furthermore, the characteristic arrow $\Delta^{\lambda}{ }_{B}$ is identified with a local Boolean cover of $\Omega$ with respect to the Boolean localization functor $\mathbf{T}$ for which the counit $\epsilon_{\Omega}$ is an isomorphism. In other words, at each Boolean algebra $B$ local subobjects of $\mathbf{M}(B)$ correspond bijectively with local Boolean covers of $\Omega$.

\section{Quantum Logical Structure of Truth Values and Criterion of Truth}

It is now important to provide an explicit representation of the elements of the quantum truth-values object $\Omega$ by calculating the colimit $\mathbf{L} \Upsilon(\mathbf{M}(-))$. In order to calculate the latter, it is necessary to specify the index or parameterizing category corresponding to the subobject functor $\Upsilon(\mathbf{M}(-))$, which is defined over the base category of Boolean event algebras $\mathcal{B}$. Following the reasoning presented in Section 5, this index category, denoted by $\int(\mathbf{\Upsilon}(\mathbf{M}(-)), \mathcal{B})$, is called the category of elements of the functor $\Upsilon(\mathbf{M}(-))$. Its objects are all pairs $(B, \lambda)$, where $\lambda$ is a subobject of $\mathbf{M}(B)$, that is a monic quantum homomorphism in $\mathbf{M}(B)$. Its morphisms are given by the arrows $(\dot{B}, \hat{\lambda}) \longrightarrow(B, \lambda)$, namely, they are those morphisms $v: \dot{B} \longrightarrow B$ of $\mathcal{B}$ for which $\lambda * v=\grave{\lambda}$, where $\lambda * v$ denotes the pullback of the subobject $\lambda$ of $\mathbf{M}(B)$ along $v$ and $\hat{\lambda}$ is a subobject of $\mathbf{M}(\dot{B})$.

Consequently, the index category corresponding to the subobject functor $\Upsilon(\mathbf{M}(-))$ is the category of its elements $\int(\mathbf{\Upsilon}(\mathbf{M}(-)), \mathcal{B})$, whence the functor $\left[\mathbf{M} \circ \int_{\Upsilon(\mathbf{M}(-))}\right]$ defines the diagram $\mathbf{X}: \mathcal{I} \rightarrow \mathcal{L}$ over which the colimit should be calculated. Hence, we obtain:

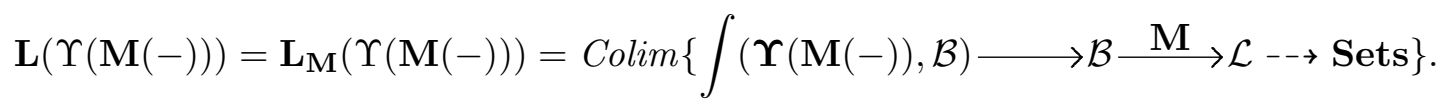

Next, we consider the set of all pairs of the form,

$$
\boldsymbol{\Sigma}=\{(\lambda, q) /(\lambda: \operatorname{Dom}(\lambda) \hookrightarrow \mathbf{M}(B)), q \in \mathbf{M}(B)\},
$$

for all Boolean algebras $B$ in $\mathcal{B}$, where $\lambda: \operatorname{Dom}(\lambda) \hookrightarrow \mathbf{M}(B)$ is a subobject of $\mathbf{M}(B)$ and $q$ is a projection operator in $\mathbf{M}(B)$. Then, from the composition law in the category of elements $\int(\mathbf{\Upsilon}(\mathbf{M}(-)), \mathcal{B})$, we obtain the relation $\top$,

$$
(\lambda * u, \dot{q}) \top(\lambda, u(\dot{q}))
$$

for any Boolean homomorphism $u: B \rightarrow B$ in the base category $\mathcal{B}$. On the basis of considerations in Section 5, it can be easily seen that the above relation induces an equivalence relation defined by the identification equations:

$$
[\lambda * v] \otimes \dot{q}=\lambda \otimes v(\dot{q}), \quad \lambda \in \Upsilon(\mathbf{M}(B)), \dot{q} \in \mathbf{M}(\dot{B}), v: \dot{B} \longrightarrow B .
$$


Furthermore, if we define $\lambda * v=\hat{\lambda}, v(\hat{q})=q$, where $\hat{\lambda}$ is a subobject of $\mathbf{M}(\dot{B})$ and $q \in \mathbf{M}(B)$, we obtain the equations:

$$
\grave{\lambda} \otimes \dot{q}=\lambda \otimes q .
$$

Thus, the set $\Upsilon(\mathbf{M}(-)) \otimes_{\mathcal{B}} \mathbf{M}$ is identified as the quotient of the set $\boldsymbol{\Sigma}$ by the equivalence relation generated by the above equations.

We conclude that the quotient set of equivalence classes of $\boldsymbol{\Sigma}$, identified with $\Upsilon(\mathbf{M}(-)) \otimes_{\mathcal{B}} \mathbf{M}$, provides the calculation of the colimit $\mathbf{L} \Upsilon(\mathbf{M}(-))$ in Sets. The tensor notation indicates that the equivalence classes of the form $\lambda \otimes q$ are canonically identified as elements of the tensor product of the functors $\Upsilon(\mathbf{M}(-))$ and $\mathbf{M}$ over the base category of Boolean event algebras $\mathcal{B}$.

It remains to show that the quotient set $\Upsilon(\mathbf{M}(-)) \otimes_{\mathcal{B}} \mathbf{M}$ has actually the structure of a quantum event algebra $\Omega$ restricted to the Boolean localization functor $\mathbf{T}$, characterized by the property that all arrows of the form $\Delta^{\lambda}{ }_{B}$ serve as local Boolean covers of $\Upsilon(\mathbf{M}(-)) \otimes_{\mathcal{B}} \mathbf{M} \equiv \Omega$ with respect to $\mathbf{T}$, for which the counit $\epsilon_{\Omega}$ is an isomorphism.

For this purpose, since the property of pullback compatibility with respect to $\mathbf{T}$ exists in $\mathcal{L}$, we may consider the arrows $h: \mathbf{M}(D) \rightarrow \mathbf{M}(B)$ and $h: \mathbf{M}(D) \rightarrow \mathbf{M}(\dot{B})$ and the following pullback diagram in $\mathcal{L}$

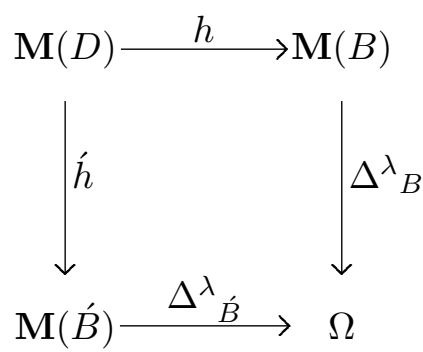

such that the relations, $h(d)=q, \hat{h}(d)=\dot{q}$ and $\lambda * h=\dot{\lambda} * \hat{h}$, are satisfied. Then, from the identification equations of the equivalence relation, we obtain:

$$
\lambda \otimes q=\lambda \otimes h(d)=[\lambda * h] \otimes d=[\hat{\lambda} * \hat{h}] \otimes d=\hat{\lambda} \otimes \dot{h}(d)=\hat{\lambda} \otimes \dot{q} .
$$

We may further define,

$$
\lambda * h=\grave{\lambda} * \hat{h}=\tau,
$$

where $\tau$ is a subobject of $\mathbf{M}(D)$. Then, apparently, it holds that:

$$
\begin{aligned}
& \lambda \otimes q=\tau \otimes d \\
& \dot{\lambda} \otimes \dot{q}=\tau \otimes d .
\end{aligned}
$$

Now, it can be easily deduced that the set $\Upsilon(\mathbf{M}(-)) \otimes_{\mathcal{B}} \mathbf{M} \equiv \Omega$ is endowed with a partial order relation with respect to $\mathbf{T}$, as follows,

$$
\lambda \otimes b \leq \rho \otimes c
$$

if and only if there exist quantum algebraic homomorphisms $\beta: \mathbf{M}(D) \rightarrow \mathbf{M}(B)$ and $\gamma$ : $\mathbf{M}(D) \rightarrow \mathbf{M}(C)$, and some $d_{1}, d_{2}$ in $\mathbf{M}(D)$, such that, $\beta\left(d_{1}\right)=b, \gamma\left(d_{2}\right)=c$, and $\lambda * \beta=$ $\rho * \gamma=\tau$. Thus, we obtain:

$$
\begin{aligned}
& \lambda \otimes b=\tau \otimes d_{1} \\
& \rho \otimes c=\tau \otimes d_{2} .
\end{aligned}
$$

We conclude that

$$
\lambda \otimes b \leq \rho \otimes c
$$

if and only if,

$$
\tau \otimes d_{1} \leq \tau \otimes d_{2} \Longleftrightarrow d_{1} \leq d_{2} .
$$


The set $\Upsilon(\mathbf{M}(-)) \otimes_{\mathcal{B}} \mathbf{M} \equiv \Omega$ may be further endowed with a maximal element which admits the following presentations:

$$
\begin{gathered}
\mathbf{1}=\tau \otimes 1:=\text { true } \quad \forall \tau \in \Upsilon(\mathbf{M}(D)) \\
\mathbf{1}=i d_{\mathbf{M}(B)} \otimes b:=\text { true } \quad \forall b \in \mathbf{M}(B),
\end{gathered}
$$

and an orthocomplementation operator,

$$
[\tau \otimes d]^{\star}=\tau \otimes d^{\star} .
$$

Then, it is easy to verify that the set $\Upsilon(\mathbf{M}(-)) \otimes_{\mathcal{B}} \mathbf{M} \equiv \Omega$ endowed with the prescribed operations is actually a quantum event algebra. Thus, $\Omega$ constitutes the classifying or truthvalues quantum event algebra, where the characteristic arrows $\Delta^{\lambda}{ }_{B}$ serve as local Boolean covers of $\Omega$ with respect to the corresponding Boolean localization functor $\mathbf{T}$. We form, therefore, the following conclusions.

Quantum Truth-Values Algebra: The elements of the quantum truth-values algebra $\Omega \equiv$ $\Upsilon(\mathbf{M}(-)) \otimes_{\mathcal{B}} \mathbf{M}$ are equivalence classes represented in tensor product form as follows:

$$
\left[\delta_{\Upsilon(\mathbf{M}(B))}\right]^{\lambda}(b):=\Delta^{\lambda}{ }_{B}(b)=\lambda \otimes b,
$$

where,

$$
[\lambda * v] \otimes \dot{b}=\lambda \otimes v(\dot{b}), \quad \lambda \in \Upsilon(\mathbf{M}(B)), \dot{b} \in \mathbf{M}(\dot{B}), v: \dot{B} \rightarrow B, v(\dot{b})=b,
$$

and $\left[\delta_{\Upsilon(\mathbf{M}(B))}\right]^{\lambda}:=\Delta^{\lambda}{ }_{B}$ denotes a local Boolean cover of $\Omega$ in the Boolean localization functor $\left[\delta_{\Upsilon(\mathbf{M}(-))}\right]^{(-)}:=\mathbf{T}$ of $\Omega$ using the unit isomorphism. Thus, truth-value assignment in quantum mechanics is localized with respect to equivalence classes of compatible Boolean frames belonging to a Boolean localization functor of a quantum event algebra.

Criterion of Truth: The criterion of truth for the category of quantum event algebras $\mathcal{L}$ with respect to a Boolean localization functor of a quantum event algebra $L$ in $\mathcal{L}$, given the preceding specification of the quantum truth-values object $\Omega$, is the following:

$$
\Delta^{\lambda}{ }_{B}(b)=\lambda \otimes b=\text { true } \quad \text { iff } \quad b \in \operatorname{Image}(\lambda), \quad \forall \lambda \in \Upsilon(\mathbf{M}(B)),
$$

where $b$ is the projection operator that identifies a corresponding quantum event or proposition $p=\psi_{B}(b) \equiv \psi_{B} \otimes b$ with respect to the local Boolean cover $\psi_{B}: \mathbf{M}(B) \rightarrow L$, i.e., with respect to the context of $\mathbf{M}(B)$.

\section{Contextual Semantics of Quantum Truth Valuation: Conceptual and Philosophical Implications}

The conceptual essence of the existence of a quantum truth-values object $\Omega$ in the category of quantum event algebras, as specified concretely in the previous section, is associated with the fact that $\Omega$ constitutes the appropriate quantum algebra for valuations of propositions describing the behavior of a quantum system, in analogy with the classical case, where the two-valued Boolean algebra 2 is properly used. Thus, in the quantum case, subobjects of a quantum event algebra $L$ admit classifying truth-values assignments in terms of characteristic morphisms, which take values not in 2 , but in the truth-values object $\Omega$ with respect to a Boolean localization functor of $L$. Intuitively, within the proposed category-theoretic scheme, the fundamental operation of the classifying object $\Omega$ is based on the following functorial aspect: a Boolean localization functor acting on a quantum event algebra $L$ induces a 
corresponding Boolean localization functor in the subobject classifier $\Omega$, such that the characteristic arrows of the subobjects of the Boolean domain covers in the former play the role of local Boolean covers in the latter. Due to the significance of this remark, let us explain the functionality of the quantum truth-values object $\Omega$ in more detail by considering the following pullback diagram

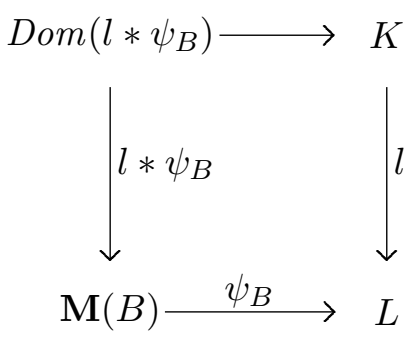

where the monic quantum homomorphism $l: K \hookrightarrow L$ denotes a subobject of a quantum event algebra $L, \psi_{B}: \mathbf{M}(B) \longrightarrow L$ signifies a local Boolean cover belonging to a Boolean localization functor of $L$, and $l * \psi_{B} \equiv \lambda$ expresses the pullback of the quantum subobject $l$ along $\psi_{B}$, thus, denoting the subobject $\lambda$ of $\mathbf{M}(B)$. According to the function of the subobject classifier $\Omega$, the characteristic arrow of the subobject $l: K \hookrightarrow L$ of $L$ is specified as an equivalence class of pullbacks of the subobject $l$ along its restrictions on all Boolean covers in a Boolean localization functor of $L$. Thus, for each projection operator $b$ in $\mathbf{M}(B)$, coordinatizing a quantum event or proposition $p=\psi_{B}(b)$ of $L$, with respect to a local Boolean cover $\psi_{B}: \mathbf{M}(B) \rightarrow L$, we obtain:

$$
l \otimes \psi_{B}(b)=\left(l * \psi_{B}\right) \otimes b=\lambda \otimes b
$$

Consequently, by using the criterion of truth of Section 8 , it is valid that $\lambda \otimes b=$ true if and only if $b$ belongs to the Image $(\lambda)$. We stress that this criterion holds for all $\lambda$ in the set of subobjects $\Upsilon(\mathbf{M}(B))$. Then, when can we say that a particular proposition $p$ in $L$ is in the Image $(l)$ ? Clearly, since each $\lambda$ is the restriction of $l$ with respect to a corresponding subobject of $\mathbf{M}(B)$, this will be the case if and only if $\lambda \otimes b=$ true for all and only those $\lambda$ for which this restriction is non-empty. Thus, for each Boolean cover $\mathbf{M}(B)$ of $L$, the value $\mathbf{1}=$ true in $\Omega$ is assigned to all those $b$ in $\mathbf{M}(B)$ belonging to the restriction of the subobject $l: K \hookrightarrow L$ of $L$ with respect to the subobject $\lambda$ of $\mathbf{M}(B)$, for all these $\lambda$. In particular, if the Boolean covers are monic morphisms, each pullback of this form is expressed as the intersection of the subobject $l$ with the corresponding cover in the Boolean localization functor.

Conceptually, every quantum event or proposition of a quantum event algebra $L$ is localized with respect to all Boolean frames $\mathbf{M}(B)$ belonging to a Boolean localization functor of $L$ by means of pulling back or restricting. Accordingly, truth-value assignment in quantum mechanics is contextualized with respect to ideals of local Boolean covers, which are pullback compatible and cover completely a quantum event algebra. With respect to each such contextualization we obtain a contextual truth valuation of the restricted proposition associated with the corresponding frame $\mathbf{M}(B)$ specified by the truth rule $\lambda \otimes b=$ true if and only if $b$ belongs to the Image $(\lambda)$, holding for every subobject $\lambda$ of $\mathbf{M}(B)$, where $b$ represents the restricted quantum proposition with respect to the Boolean frame $\mathbf{M}(B)$. In this sense, elementary propositions associated with the description of the behavior of a quantum system in various contexts of observation, identified by local frames in Boolean localization functors of a quantum event algebra, are naturally assigned truth values in $\Omega$ according to the preceding criterion of truth.

We emphasize in this respect that the quantum truth-values object $\Omega$ enables not only a determinate truth valuation in each fixed frame $\mathbf{M}(B)$, but in addition, it amalgamates internally all compatible truth valuations with respect to all Boolean frames belonging to a 
Boolean localization functor of $L$. This, in effect, is established by the formation of equivalence classes, which are represented in tensor product form, via the truth-value true in $\Omega$. The maximally compatible equivalence class identified with the maximal element true in $\Omega$ specifies a complete description of states of affairs with respect to the considered Boolean localization functor. In this way, truth-value assignment in quantum mechanics is localized and consequently contextualized with respect to tensor product equivalence classes formed among compatible Boolean frames belonging to a Boolean localization functor of a quantum event algebra.

Importantly, the attribution of truth values to quantum mechanical propositions arising out of the preceding category-theoretic scheme circumvents consistently the semantic ambiguity with respect to binary truth-value assignments to propositions that is inherent in conventional quantum mechanics, in the following sense. All propositions that are certainly true or certainly false (assigned probability value 1 or 0 ) according to conventional quantum mechanics are also certainly true or certainly false according to the category-theoretic approach. The remaining propositions (assigned probability value different from 1 and 0 ) are semantically undecidable according to the former interpretation, they are neither true nor false, while they have determinate (albeit unknown) truth values, they are either true or false, according to the latter. These values, however, depend not only on the state of the physical system that is considered but also on the context through which the system is investigated, thus capturing the endemic feature of quantum contextuality. Indeed, as already explained, the existence of the subobject classifier $\Omega$ leads naturally to contextual truth-value assignments to quantum mechanical propositions, where each proposition pertaining to a physical system under investigation acquires a determinate truth value with respect to the context defined by the corresponding observable to be measured.

It is instructive to note at this point that considering a preparatory Boolean environment for a system to interact with a measuring arrangement does not determine which event will take place, but it does determine the kind of event that will take place. It forces the outcome, whatever it is, to belong to a certain definite Boolean subalgebra of events for which the standard measurement conditions are invariant. Such a set of standard conditions for a definite kind of measurement constitutes a set of necessary and sufficient constraints for the occurrence of an event of the selected kind. As already explained in Section 4, this equivalently means in the light of our approach that a Boolean algebra in the lattice of quantum events picked by an observable to be measured instantiates locally a physical context, which may serve as a logical Boolean reference frame relative to which results of measurement are being coordinatized. In this respect, Boolean frames or instances of concrete experimental arrangements in quantum mechanics play a role analogous to the reference frames of rods and clocks in relativity theory in establishing a perspectival aspect to reality. We may further observe that the variation of the base Boolean event algebras in the proposed category-theoretic scheme is actually arising from any experimental praxis aiming to fix or prepare the state of a quantum system and corresponds, in this sense, to the variation of all possible Boolean preparatory contexts pertaining to a quantum system for extracting information about it. The key philosophical meaning of this approach implies, therefore, the view that the quantum world is comprehended through overlapping Boolean frames, which interlock, in a category-theoretical environment, to form a coherent picture of the whole in a nontrivial way. Most importantly, this viewpoint is formalized categorically as an instance of the concept of the adjunction of our interpretative scheme between the category of (pre)sheaves of variable local Boolean frames and the category of quantum event algebras. In this way, the global structural information of a quantum event algebra can be captured homomorphically or, by restriction to a Boolean localization functor, can be completely constituted (up to isomorphism) by means of gluing together the information collected in all compatible Boolean frames in the form of appropriate equivalence classes (Zafiris \& Karakostas 2013). Moreover, as has been shown, the Boolean frames-quantum adjunction provides the conceptual and technical means to establish that 
the category of quantum event algebras is equipped with a quantum truth-values object $\Omega$, a classifying object, which generalizes the classical binary object by classifying information in terms of contextual truth valuations with respect to these distinct Boolean frames.

In view of the preceding considerations, therefore, and in relation to philosophical matters, we propose a contextual account of truth that is compatible with the propositional structure of quantum theory by conforming to the following instance of the correspondence scheme:

[CC] The proposition that $P$-in- $C$ is true if and only if there is a state of affairs $X$ such that (1) $P$ expresses $X$ in $C$ and (2) $X$ obtains,

where $C$ denotes, in general, the context of discourse, and specifically, in relation to the aforementioned quantum mechanical considerations, the experimental context $C_{A}(\mathbf{M})$, linked to the proposition $P$ under investigation, that is associated with a particular Boolean frame $\mathbf{M}(B)$ belonging to a Boolean localization functor of $L$, and $A$ indicating the physical magnitude under investigation.

Let us initially note that the proposed contextual account of truth satisfies Tarski's (1935/1956, p. 188) criterion of material adequacy, known as "convention T" or "schema T", for a theory of truth:

(T) The proposition that " $P$ " is true if, and only if, $P$,

where the symbol " $P$ " in $(\mathrm{T})$ represents the name of the proposition which $P$ stands for. To this purpose, let us consider a particular proposition $P$ : "system $S$ has the property $P(A)$ ". Assume context-dependence with regard to $P(A)$, i.e., the latter property of $S$ holds within context $C$. Then, proposition $P$ is concisely translated as: "system $S$ has the property $P(A)$ in- $C$ ". Suppose now, harmlessly, that this proposition is true. If so, the following instance of the T-schema must be true a priori:

The proposition that "system $S$ has the property $P(A)$-in- $C$ " is true if, and only if, system $S$ has the property $P(A)$-in- $C$.

If, however, the property $P(A)$ of $S$ is context-dependent upon $C$, then the proposition that system $S$ has the property $P(A)$ must also be context-dependent upon $C$. Thus, in conformity with the propositional status entering into the scheme $[\mathrm{CC}]$, the preceding instance of the $\mathrm{T}$ schema can be written equivalently in succinct form as:

The proposition that " $P$-in- $C$ " is true if, and only if, $P$-in- $C$.

Clearly, the logical operation of the bi-conditional in the preceding T-sentence is governed again by the T-schema, or the truism, that the content of a proposition determines the necessary and sufficient conditions under which it is true. Thus for any given true proposition which is context-dependent upon $C$, the fact that makes it true is the context-dependent fact (or state of affairs) upon $C$ that the proposition expresses. Truth contextuality follows naturally from the contextuality of makers of propositional truths. If the latter are contextdependent, then whatever truths may be expressed about them must also be contextual.

The proposed account of truth, as encapsulated by the scheme [CC] of contextual correspondence, incorporates explicitly a context-dependence texture of the "world-word" relation, if the world, in its microphysical dimension, is to be correctly describable. The truth-making relationship is now established, not in terms of a raw un-conceptualized reality, as envisaged by the traditional scheme of correspondence truth, but between a well-defined portion of reality as carved out by the experimental context and the propositional content that refers to the selected context. Such interdependence of propositional content and referential context is not by virtue of some meta-scientific principle or philosophical predilection, but by virtue of the microphysical nature of physical reality displaying a context-dependence of facts. On the 
other hand, the traditional conception of correspondence truth, as exemplified either by the alethic scheme $[\mathrm{CF}]$ or $[\mathrm{CS}]$, alluded to in the introduction, and involving a direct contextindependent relation between singular terms of propositions and definite autonomous facts of an external reality, may be viewed as a species or as a limit case of the more generic alethic scheme of contextual correspondence [CC], when the latter is applied in straightforward unproblematic circumstances where the non-explicit specification of a context of discourse poses no further consequences.

If, however, ascriptions of truth values to propositions are context-dependent in some way as the scheme $[\mathrm{CC}]$ implies, it would appear, according to traditional thinking, that one is committed to antirealism about truth. In our opinion, this assumption is mistaken. The contextual account of truth suggested here conforms to a realist conception of truth, which, moreover, is compatible with contemporary physics. Such an account essentially denies that there can be a "God's-eye view" or an absolute Archimedean standpoint from which to state the totality of facts of nature. For, in virtue of the Kochen-Specker theorem, there simply does not exist, within a quantum mechanical discourse, a consistent binary assignment of determinately true or determinately false propositions independent of the appeal to a context. Propositional content seems to be linked to a context. This connection between referential context and propositional content means that a descriptive elementary proposition in the domain of quantum mechanics is, in a sense, incomplete unless it is accompanied by the specified conditions of an experimental context under which the proposition becomes effectively truth-valued (see, in addition, Karakostas 2014). In view of our approach, the latter observation underlines the fact that the conceptual significance of a logic of propositions referring to the description of a quantum system lies at the level of contextual propositions holding in distinct Boolean frames. The proposed categorical framework reveals precisely that the logic of a quantum event structure is to be sought not in its internal constitution as a set-theoretical entity endowed with unrestricted primary qualities, but rather, in the form of its relationship with the Boolean kind of structure through the established network of adjoint functors between the topos of variable overlapping Boolean frames and the category of quantum event algebras. This conception enlightens yet further the connection between a quantum algebra of events and its underlying building blocks of Boolean algebras by clarifying the contextual character of quantum theory.

We note in this respect that the proposed account of truth of contextual correspondence $[\mathrm{CC}]$ ought to be disassociated from a pragmatic instrumental notion of truth. From the category theoretical perspective of the present paper, the reference to a Boolean preparatory experimental context should not be viewed primarily as offering the evidential or verificationist basis for the truth of a proposition; it does not aim to equate truth to verification. Nor should it be associated with practices of instrumentalism, operationalism and the like; it does not aim to reduce theoretical terms to products of operational procedures. It rather provides the appropriate conditions under which it is possible for a proposition to receive consistently a truth value. Whereas in classical mechanics the conditions under which elementary propositions are claimed to be true or false are determinate independently of the context in which they are expressed, in contradistinction, the truth-conditions of quantum mechanical propositions are determinate within a context. In other words, the specification of the context is part and parcel of the truth-conditions that should obtain for a proposition in order the latter to be invested with a determinate (albeit unknown) truth value. Otherwise, the proposition is, in general, semantically undecidable. In the quantum description, therefore, the introduction of the experimental context is to select at any time $t$ a specific complete Boolean subalgebra $\mathbf{M}\left(B_{A}\right)$ in the global non-Boolean algebra of propositions of a quantum system as co-definite; that is, each proposition in $\mathbf{M}\left(B_{A}\right)$ is assigned at time $t$ a definite truth value, "true" or "false", or equivalently, each corresponding property of the system either obtains or does not obtain. Significantly, due to the functioning of the quantum classifying object $\Omega$, as specified by the explicit criterion of Section 8, the truth of a proposition in a Boolean frame remains 
invariant with respect to truth valuations of all other compatible propositions in all Boolean frames $\mathbf{M}(B)$ belonging to a Boolean localization functor of a quantum event algebra. Thus, in our approach, the specification of a particular Boolean frame, or a concrete experimental context, provides in a consistent manner the necessary conditions whereby bivalent assignment of truth values to quantum mechanical propositions is in principle applicable. This marks the fundamental difference between conditions for well-defined attribution of truth values to propositions and mere verification conditions.

This element also signifies the transition from the transcendence condition of the conventional correspondence theory of truth of Section 1 to a reflective-like transcendental reasoning ${ }^{2}$ of the proposed account of truth. That is, it signifies the transition from the uncritical qualification of truth values to propositions beyond the limits of experience and acknowledging them as being true or false simpliciter, to the demarcation of the limits of possible experience or to the establishment of pre-conditions which make possible the attribution of truth values to propositions. In the quantum description, therefore, the specification of the experimental context forms a pre-condition of quantum physical experience, which is necessary if quantum mechanics is to grasp empirical reality at all. Any microphysical fact or event that 'happens' is raised at the empirical level only in conjunction with the specification of an experimental context that conforms to a set of observables co-measurable by that context. The introduction of the experimental context furnishes thus the status of a material presupposition of any empirical access to the quantum level of reality, of any possible empirical inquiry on the microscopic scale, and hence of any possible cognizance of microphysical objects as scientific objects of experience. In this respect, the specification of the context constitutes a methodological act preceding any empirical truth in the quantum domain and making it possible.

Nor the proposed contextual conception of truth is a relative notion itself; the propositions to which it applies are relative. They are relative to a specific Boolean subalgebra of propositions which are determinately true or false of a system at any particular time. For, as already argued, a quantum mechanical proposition is not true or false simpliciter, but acquires a determinate truth value with respect to a well-defined context of discourse as specified by the state of the quantum system concerned and a particular observable to be measured. Thus, the conditions under which a proposition is true are jointly determined by the context in which the proposition is expressed and the actual microphysical state of affairs as projected into the specified context. What makes a proposition true, therefore, is not that is relative to the context (as an alethic relativist must hold, see, for instance, MacFarlane 2005) but whether or not the conditions in question obtain. The obtainment of the conditions implies that it is possible for us to make, in an overall consistent manner, meaningful statements that the properties attributed to quantum objects are part of physical reality. In our approach, therefore, the reason that a proposition is true is because it designates an objectively existing state of affairs, albeit of a contextual nature. In relation to the latter, we wish to emphasize the fact that, in contrast to a panoptical "view from nowhere" of the classical paradigm, the general epistemological implication of quantum theory acknowledges in an essential way a contextual character of knowledge. The classical idea that one can reasonably talk about "all entities", as if the terms "entity" or "object" had a unique, fixed meaning, independently of the appeal to a particular context of reference, is inadequate in the microphysical level of discourse. Whereas in the old classical paradigm, reality was conceived as an absolute concept totally independent of the process of knowledge, in the new quantum paradigm, epistemology

\footnotetext{
${ }^{2}$ Transcendental reasoning is understood in contemporary terms as the method of inquiry seeking to investigate the necessary conditions or presuppositions for the possibility of some given actuality from within the sphere constrained by those conditions. A genuinely transcendental approach, therefore, operates reflectively from within our worldly conditions, standards and practices in order to examine the preconditions and limits of experience or knowledge, thus opposing any supposedly transcendent, non-perspectival, metaphysically fixed point of reference. A collection of essays focusing on the broader framework of transcendental inquiry, both in its historical context as well as its more recent appearance in philosophizing, has been edited by Malpas (2003).
} 
- namely, the understanding of the process of knowing - has to be explicitly included in the description of natural phenomena. Epistemology necessarily becomes now an integral part of the theory, an issue pointing at the same time at the meta-theoretical, philosophical level towards an interconnection among epistemological and ontological considerations.

\section{Appendix}

Category theory provides a general theoretical framework for dealing with systems, formalized through appropriate mathematical structures, by putting the emphasis on their mutual relations and transformations. The central focus of the categorical way of rethinking basic notions can be described as a shift in the emphasis of what is considered to be fundamental for the formation of structures. In the set-theoretic mode of thinking, structures of any conceivable form are defined as sets of elements endowed with appropriate relations. In the category-theoretic mode, the emphasis is placed on the transformations among the objects of a category and the processes preserving them by means of appropriate structural constraints on the collection of these transformations. In this sense, the notion of structure does not refer exclusively to a fixed universe of sets of pre-determined elements, but acquires a variable reference. The basic categorical notions used systematically in the present paper are summarized as follows.

Categories : A category $\mathcal{C}$ is an aggregate consisting of the following:

1. A class $O b(\mathcal{C})$, whose elements $A, B, \ldots$ are called objects. For each object $A$ an element $i d_{A}: A \rightarrow A$ is distinguished; it is called the identity morphism for $A$.

2. A class $\operatorname{Hom}(\mathcal{C})$, whose elements $f, g, \ldots$ are called morphisms or arrows. Each morphism $f: A \rightarrow B$ is associated with a pair of objects, known as its domain and codomain respectively. Note that in category theory this arrow notation is used even if $f$ is not a function in the normal set-theoretic sense. The expression $\operatorname{Hom}_{\mathcal{C}}(A, B)$ denotes the $H o m$-class of all morphisms from $A$ to $B$.

3. A binary operation $\circ$, called composition of morphisms, such that, for given arrows $f: A \rightarrow B$ and $g: B \rightarrow E$, that is, with codomain of $f$ equal to the domain of $g$, then $f$ and $g$ can be composed to give an arrow $g \circ f: A \rightarrow E$. Generally, for any three objects $A, B$, and $E$ in $\mathcal{C}$ the set mapping is defined as:

$$
\operatorname{Hom}_{\mathcal{C}}(B, E) \times \operatorname{Hom}_{\mathcal{C}}(A, B) \rightarrow \operatorname{Hom}_{\mathcal{C}}(A, E) .
$$

The operation of composition is associative, $h \circ(g \circ f)=(h \circ g) \circ f$, for all $f: A \rightarrow B$, $g: B \rightarrow E, h: E \rightarrow D$, satisfying also the property of identity, $f \circ i d_{A}=f=i d_{B} \circ f$, for all $f: A \rightarrow B$.

For an arbitrary category $\mathcal{C}$ the opposite category $\mathcal{C}^{o p}$ is defined in the following way. The objects are the same, but $\operatorname{Hom}_{\mathcal{C}^{o p}}(A, B)=\operatorname{Hom}_{\mathcal{C}}(B, A)$, namely, all arrows are inverted. A category $\mathcal{C}$ is called small if the classes of its objects and morphisms form genuine sets.

Functors : Let $\mathcal{C}, \mathcal{D}$ be categories. A covariant functor $\mathbf{F}: \mathcal{C} \rightarrow \mathcal{D}$ is a class mapping that:

1. Associates to each object $A \in \mathcal{C}$ an object $\mathbf{F}(A) \in \mathcal{D}$.

2. Associates to each morphism $f: A \rightarrow B \in \mathcal{C}$ a morphism $\mathbf{F}(f): \mathbf{F}(A) \rightarrow \mathbf{F}(B) \in \mathcal{D}$. 
3. Makes these assignments by preserving identity morphisms and compositions, i.e., $\mathbf{F}\left(i d_{A}\right)=$ $i d_{\mathbf{F}(A)}$, and $\mathbf{F}(g \circ f)=\mathbf{F}(g) \circ \mathbf{F}(f)$.

A contravariant functor $\hat{\mathbf{F}}: \mathcal{C} \rightarrow \mathcal{D}$ is, by definition, a covariant functor $\mathbf{F}: \mathcal{C}^{o p} \rightarrow \mathcal{D}$. A functor, therefore, is a type of mapping between categories that associates to every object of one category an object of another category and to every morphism in the first category a morphism in the second by preserving domains and codomains, identity morphisms, and compositions. A functor $\mathbf{F}: \mathcal{C} \rightarrow \mathcal{D}$ thus gives a sort of 'picture' of category $\mathcal{C}$ in $\mathcal{D}$ by preserving the structure of $\mathcal{C}$.

Natural Transformations : $\quad$ Let $\mathcal{C}, \mathcal{D}$ be categories, and let further $\mathbf{F}, \mathbf{G}$ be functors from the category $\mathcal{C}$ to the category $\mathcal{D}$. A natural transformation $\tau: \mathbf{F} \rightarrow \mathbf{G}$ is a mapping that assigns to each object $A$ in $\mathcal{C}$ a morphism $\tau_{A}: \mathbf{F}(A) \rightarrow \mathbf{G}(A)$ in $\mathcal{D}$, called the component of $\tau$ at $A$, such that for every arrow $f: A \rightarrow B$ in $\mathcal{C}$ the following diagram in $\mathcal{D}$ commutes:

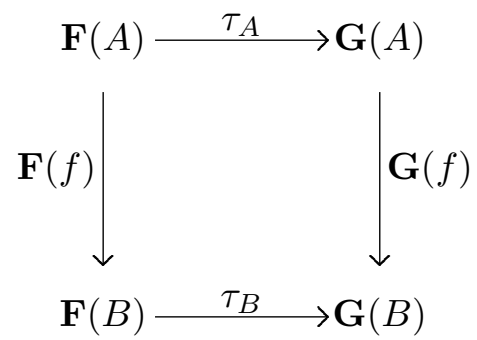

That is, for every arrow $f: A \rightarrow B$ in $\mathcal{C}$, we have:

$$
\mathbf{G}(f) \circ \tau_{A}=\tau_{B} \circ \mathbf{F}(f) .
$$

Thus, natural transformations define structure preserving mappings of functors. Pictorially, one can think of this situation as follows: if the parallel functors $\mathbf{F}: \mathcal{C} \rightarrow \mathcal{D}$ and $\mathbf{G}: \mathcal{C} \rightarrow \mathcal{D}$ are thought of as projecting a 'picture' of category $\mathcal{C}$ in $\mathcal{D}$, then a natural transformation is a way to transform globally or systematically the 'picture' defined by $\mathbf{F}$ onto the 'picture' defined by $\mathbf{G}$.

Natural Isomorphisms : A natural transformation $\tau: \mathbf{F} \rightarrow \mathbf{G}$ is called a natural isomorphism if every component $\tau_{A}$ is invertible.

Adjoint Functors : Let $\mathbf{F}: \mathcal{C} \rightarrow \mathcal{D}$ and $\mathbf{G}: \mathcal{D} \rightarrow \mathcal{C}$ be functors. We say that $\mathbf{F}$ is left adjoint to $\mathbf{G}$ (correspondingly, $\mathbf{G}$ is right adjoint to $\mathbf{F}$ ), if there exists a bijective correspondence between the arrows $\mathbf{F}(C) \rightarrow D$ in $\mathcal{D}$ and $C \rightarrow \mathbf{G}(D)$ in $\mathcal{C}$, which is natural in both $C$ and $D$ :

$$
\mathbf{F}: \mathcal{C} \longleftrightarrow \mathcal{D}: \mathbf{G}
$$

This means that the objects of the categories $\mathcal{C}$ and $\mathcal{D}$ are related with each other through natural transformations. Then, the above pair of adjoint functors constitutes a categorical adjunction. The latter concept is of fundamental logical and mathematical importance expressing a generalization of equivalence of categories.

Diagrams : A diagram $\mathbf{X}=\left(\left\{X_{i}\right\}_{i \in I},\left\{F_{i j}\right\}_{i, j \in I}\right)$ in a category $\mathcal{C}$ is defined as an indexed family of objects $\left\{X_{i}\right\}_{i \in I}$ and a family of morphisms sets $\left\{F_{i j}\right\}_{i, j \in I} \subseteq \operatorname{Hom}_{\mathcal{C}}\left(X_{i}, X_{j}\right)$. 
Cocones : A cocone of the diagram $\mathbf{X}=\left(\left\{X_{i}\right\}_{i \in I},\left\{F_{i j}\right\}_{i, j \in I}\right)$ in a category $\mathcal{C}$ consists of an object $X$ in $\mathcal{C}$, and for every $i \in I$, a morphism $f_{i}: X_{i} \rightarrow X$, such that $f_{i}=f_{j} \circ f_{i j}$ for all $j \in I$, that is, for every $i, j \in I$, and for every $f_{i j} \in F_{i j}$ the diagram below commutes:

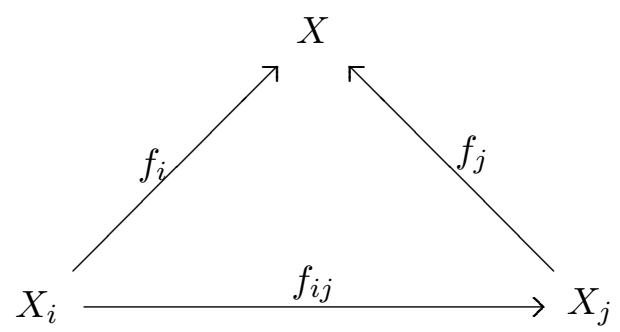

Colimits : A colimit of the diagram $\mathbf{X}=\left(\left\{X_{i}\right\}_{i \in I},\left\{F_{i j}\right\}_{i, j \in I}\right)$ is a cocone with the property that for every other cocone given by morphisms $f_{\dot{i}}: X_{i} \rightarrow \dot{X}$, there exists exactly one morphism $f: X \rightarrow \dot{X}$, such that $f_{\hat{i}}=f \circ f_{i}$, for all $i \in I$ (universality property).

Reversing the arrows in the above definitions of cocone and colimit of a diagram $\mathbf{X}=$ $\left(\left\{X_{i}\right\}_{i \in I},\left\{F_{i j}\right\}_{i, j \in I}\right)$ in a category $\mathcal{C}$ results in the dual notions called cone and limit of $\mathbf{X}$, respectively.

Pullbacks: Let $f: A \rightarrow C$ and $g: B \rightarrow C$ be morphisms with common codomain in a category $\mathcal{C}$. A pullback of morphisms $f$ and $g$ is a triple $\left(P, p_{1}, p_{2}\right)$ consisting of an object $P$ and morphisms $p_{1}: P \rightarrow A$ and $p_{2}: P \rightarrow B$, in $\mathcal{C}$, such that $f \circ p_{1}=g \circ p_{2}$, i.e., the following diagram commutes:

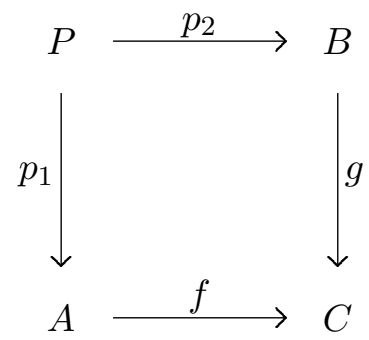

In addition, the pullback $\left(P, p_{1}, p_{2}\right)$ must be universal with respect to this diagram. That is, for every other such triple $\left(Q, q_{1}, q_{2}\right)$ in $\mathcal{C}$, there exists a unique morphism $u: Q \rightarrow P$ such that $q_{1}=p_{1} \circ u$ and $q_{2}=p_{2} \circ u$. As with all categorical universal constructions, the pullback of two morphisms, if it exists, is uniquely defined up to an isomorphism. The dual notion of a pullback is that of a pushout.

Subobject Functors : A subobject functor Sub : $\mathcal{C}^{o p} \rightarrow$ Sets in any category $\mathcal{C}$ with finite limits is constructed as follows:

1. For an object $A$ of $\mathcal{C}, \operatorname{Sub}(A)$ is the set of equivalence classes of subobjects of $A$, i.e., the set of equivalence classes of monic arrows $\mu: M \rightarrow A$.

2. Given a morphism $f: B \rightarrow A$ and a monic arrow $\mu: M \rightarrow A, \mathbf{S u b}(f): \operatorname{Sub}(A) \rightarrow$ $\operatorname{Sub}(B)$ is obtained by pulling back $\mu$ along $f$ as in the following diagram:

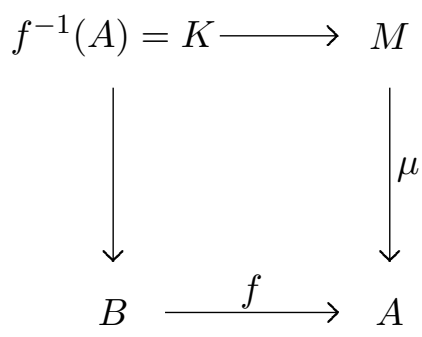


Sieves : A sieve on an object $A$ of a category $\mathcal{C}$ is a collection $S$ of morphisms with codomain $A$ in $\mathcal{C}$ such that, if $f: B \rightarrow A$ belongs to $S$ and $g: C \rightarrow B$ is any morphism, then $f \circ g: C \rightarrow A$ belongs also to $S$.

Generalized Covering Systems : A generalized topological covering system (or a localization system) on a category $\mathcal{C}$ is a function $\mathbf{J}$ which assigns to each object $A$ of $\mathcal{C}$ a collection $\mathbf{J}(A)$ of sieves on $A$, called covering $A$-sieves, such that:

1. For each $\mathcal{C}$-object $A$, the maximal $A$-sieve $\{f: \operatorname{cod}(f)=A\}$ belongs to $\mathbf{J}(A)$ (maximality condition).

2. If $S$ belongs to $\mathbf{J}(A)$, then, for any arrow $h: C \rightarrow A$, the pullback sieve $h^{*}(S)=\{g$ : $D \rightarrow C,(h \circ g) \in S\}$, for any object $D$ of $\mathcal{C}$, belongs to $\mathbf{J}(C)$ (stability condition).

3. If $S$ belongs to $\mathbf{J}(A)$, and if for each arrow $h: C_{h} \rightarrow A$ in $S$ there is a sieve $R_{h}$ belonging to $\mathbf{J}\left(C_{h}\right)$, then the set of all composites $h \circ f$, with $h \in S$ and $f \in R_{h}$, belongs to $\mathbf{J}(A)$ (transitivity condition).

A generalized topological covering system on $\mathcal{C}$, satisfying the previously stated conditions, is equivalent to the notion of a Grothendieck topology on $\mathcal{C}$. If $\mathcal{C}$ is a (small) category equipped with a Grothendieck topology $\mathbf{J}$, then the pair $(\mathcal{C}, \mathbf{J})$ defines a site.

Presheaves : A presheaf $\mathbf{P}$ on a small category $\mathcal{C}$ is a function that:

1. Assigns to each $\mathcal{C}$-object $A$ a set $\mathbf{P}(A)$.

2. Assigns to each $\mathcal{C}$-morphism $f: B \rightarrow A$ a set function, $\mathbf{P}(f): \mathbf{P}(A) \rightarrow \mathbf{P}(B)$.

3. Makes these assignments by preserving identity morphisms and compositions, i.e., $\mathbf{P}\left(i d_{A}\right)=$ $i d_{\mathbf{P}(A)}$; and, if $g: C \rightarrow B$ and $f: B \rightarrow A$, then $\mathbf{P}(f \circ g)=\mathbf{P}(g) \circ \mathbf{P}(f)$.

Intuitively, a presheaf is a collection of sets that vary in a 'meshing' way between objects $A, B, \ldots$ of the category $\mathcal{C}$. In terms of contravariant and covariant functors, a presheaf on $\mathcal{C}$ is a contravariant functor from category $\mathcal{C}$ to the category Sets of normal sets. Equivalently, it is a covariant functor from the opposite category $\mathcal{C}^{o p}$ to Sets. The collection of all presheaves on $\mathcal{C}$ forms a category, denoted Sets $^{\mathcal{C}^{o p}}$, in which morphisms between presheaves $\mathbf{P}$ and $\mathbf{Q}$ are natural transformations $\tau: \mathbf{P} \rightarrow \mathbf{Q}$.

Sheaves : Given a site $(\mathcal{C}, \mathbf{J})$, a sheaf is defined to be a presheaf $\mathbf{P}$ such that for all objects $A$ of $C$ and all covering sieves $S$ on $A$, any natural transformation $\tau: S \rightarrow \mathbf{P}$ has a unique extension to $\operatorname{Hom}(-, A)$, that is there is an isomorphism between the set of natural transformations induced by the inclusion $S \mapsto \operatorname{Hom}(-, A)$,

$$
\operatorname{Hom}(S, \mathbf{P}) \cong \operatorname{Hom}(\operatorname{Hom}(-, A), \mathbf{P}) .
$$

Observe that a sheaf is systematically connected to the covering sieves of the objects of $C$. Its most characteristic property is that it provides a unique way to "glue" together functions that are defined locally. In this respect, through the notion of a sheaf, one can systematically move from the local level to the global, or inversely, the global can be constructed from the local in a systematic manner. 
Topoi : A topos, $\mathcal{T}$, is a type of a category that is equivalent to the category of sheaves of sets on a topological space, or more generally, on a site. For the objects of a topos, which are sheaves of sets, the usual constructions of the category, Sets, of sets can be defined in a generalized categorical manner. For this reason topoi may serve as alternative models of set theory, especially, in view of the fact that each topos completely defines its own mathematical framework. The most important properties of a topos, $\mathcal{T}$, include the following:

1. There is an initial object $0_{\mathcal{T}}$ in $\mathcal{T}$ such that, for any object $A$ in the topos, there is a unique arrow $0_{\mathcal{T}} \rightarrow A$. It is the analogue of the empty set, $\emptyset$.

2. There is a terminal object $1_{\mathcal{T}}$ in $\mathcal{T}$ such that, for any object $A$ in the topos, there is a unique arrow $A \rightarrow 1_{\mathcal{T}}$. It is the analogue of the singleton set $\{*\}$.

For any object $A$ in the topos, an arrow $1_{\mathcal{T}} \rightarrow A$ is called a global element of $\mathrm{A}$.

3. For any objects $A, B$ in $\mathcal{T}$, there is a product $A \times B$ in the topos. It is the analogue of the Cartesian product in set theory. A topos always has pull-backs, and the product is just a special case of this.

For any objects $A, B$ in $\mathcal{T}$, there is a co-product $A \sqcup B$ in the topos. It is the analogue of the disjoint union in set theory. A topos always has push-outs, and the co-product is just a special case of this.

4. There is exponentiation, associating to each pair of objects $A, B$ in $\mathcal{T}$ an object $A^{B}$, which is the topos analogue of the set of functions $f: B \rightarrow A$ between sets $B$ and $A$ in set theory. The characteristic property of exponentiation is that, given any object $X$, there is an isomorphism

$$
\operatorname{Hom}_{\mathcal{T}}\left(X, A^{B}\right) \cong \operatorname{Hom}_{\mathcal{T}}(X \times B, A)
$$

that is natural in $A$ and $X$. In set theory, the relevant statement is that a parameterized family of functions $\chi \mapsto f_{\chi}: A \rightarrow B, \chi \in X$, is equivalent to a single function $F$ : $X \times A \rightarrow B$ defined by $F(\chi, \alpha):=f_{\chi}(\alpha)$ for all $\chi \in X, \alpha \in A$.

5. There is a subobject classifier $\Omega_{\mathcal{T}}$.

\section{References}

1. Abramsky, S., \& Brandenburger, A. (2011). The sheaf-theoretic structure of non-locality and contextuality. New Journal of Physics, 13, 113036.

2. Awodey, S. (2010). Category theory (2nd ed.). Oxford: Oxford University Press.

3. Bell, J.L. (1988/2008). Toposes and local set theories. New York: Dover.

4. Berg, B. v.d., \& Heunen, C. (2012). Noncommutativity as a colimit. Applied Categorical Structures, 20(4), 393-414.

5. Blackburn, S., \& Simmons K. (1999). Truth (Oxford Readings in Philosophy). Oxford: Oxford University Press.

6. Burgess, A., \& Burgess, J.P. (2011). Truth (Princeton Foundations of Contemporary Philosophy). Princeton: Princeton University Press.

7. Coecke, B. (2010). Quantum picturalism. Contemporary Physics, 51(1), 59-83.

8. Dalla Chiara, M., Giuntini, R., \& Greechie, R. (2004). Reasoning in quantum theory. Dordrecht: Kluwer. 
9. Davis, M. (1977). A relativity principle in quantum mechanics. International Journal of Theoretical Physics, 16, 867-874.

10. Devitt, M. (2001). The metaphysics of truth. In M. Lynch (ed.), The nature of truth: Classic and contemporary perspectives (pp. 579-611). Cambridge MA: MIT Press.

11. Dirac, P.A.M. (1958). Quantum mechanics (4th ed.). Oxford: Clarendon Press.

12. Domenech, G., \& Freytes, H. (2005). Contextual logic for quantum systems. Journal of Mathematical Physics, 46, 012102.

13. Döring, A., \& Isham, C.J. (2011). "What is a thing?": Topos theory in the foundations of physics. In B. Coecke (ed.), New structures for physics, Lecture Notes in Physics, vol. 813 (pp. 753-937). Berlin: Springer.

14. Epperson, M., \& Zafiris, E. (2013). Foundations of relational realism: A topological approach to quantum mechanics and the philosophy of nature. Book Series: Contemporary Whitehead Studies. New York: Lexington Books.

15. Goldblatt, R. (1984/2006). Topoi: The categorial analysis of logic (rev. 2nd ed.). New York: Dover.

16. Heunen, C., Landsman, N.P., \& Spitters, B. (2011). Bohrification. In H. Halvorson (ed.), Deep beauty (pp. 271-313). New York: Cambridge University Press.

17. Isham, C.J., \& Butterfield, J. (1998). A topos perspective on the Kochen-Specker theorem: I. Quantum states as generalized valuations. International Journal of Theoretical Physics, 37, 2669-2733.

18. Karakostas, V. (2007). Nonseparability, potentiality, and the context-dependence of quantum objects. Journal for General Philosophy of Science, 38, 279-297.

19. Karakostas, V. (2012). Realism and objectivism in quantum mechanics. Journal for General Philosophy of Science, 43, 45-65.

20. Karakostas, V. (2014). Correspondence truth and quantum mechanics. Axiomathes, 24, 343358.

21. Kochen, S., \& Specker, E.P. (1967). The problem of hidden variables in quantum mechanics. Journal of Mathematics and Mechanics, 17, 59-87.

22. Lawvere, W., \& Schanuel, S. (2009). Conceptual mathematics (2nd ed.). Cambridge: Cambridge University Press.

23. MacFarlane, J. (2005). Making sense of relative truth. Proceedings of the Aristotelian Society, March 15, 321-339.

24. MacLane, S., \& Moerdijk, I. (1992). Sheaves in geometry and logic. New York: Springer.

25. Malpas, J. (ed.) (2003). From Kant to Davidson: Philosophy and the idea of the transcendental. London \& New York: Routledge.

26. Rédei, M. (1998). Quantum logic in algebraic approach. Dordrecht: Kluwer.

27. Svozil, K. (1998). Quantum logic. Singapore: Springer.

28. Takeuti, G. (1978). Two applications of logic to mathematics. Part I: Boolean valued analysis. Publications of Mathematical Society of Japan 13. Tokyo and Princeton: Iwanami and Princeton University Press.

29. Tarski, A. (1935/1956). The concept of truth in formalized languages. In A. Tarski, Logic, semantics, metamathematics: Papers from 1923 to 1938 (pp. 152-278). Oxford: Clarendon Press.

30. Von Neumann, J. (1955). Mathematical foundations of quantum mechanics. Princeton: Princeton University Press.

31. Zafiris, E. (2004). Boolean coverings of quantum observable structure: A setting for an abstract differential geometric mechanism. Journal of Geometry and Physics, 50, 99-114.

32. Zafiris, E. (2006a). Generalized topological covering systems on quantum events structures. Journal of Physics A: Mathematical and General, 39, 1485-1505. 
33. Zafiris, E. (2006b). Sheaf-theoretic representation of quantum measure algebras. Journal of Mathematical Physics, 47, 092103.

34. Zafiris, E., \& Karakostas, V. (2013). A categorial semantic representation of quantum event structures. Foundations of Physics, 43, 1090-1123. 